\title{
Probability, coherent belief and coherent belief changes
}

\author{
John Cantwell ${ }^{1,2} \cdot$ Hans Rott ${ }^{2,3}$
}

Published online: 26 July 2019

(C) The Author(s) 2019

\begin{abstract}
This paper is about the statics and dynamics of belief states that are represented by pairs consisting of an agent's credences (represented by a subjective probability measure) and her categorical beliefs (represented by a set of possible worlds). Regarding the static side, we argue that the latter proposition should be coherent with respect to the probability measure and that its probability should reach a certain threshold value. On the dynamic side, we advocate Jeffrey conditionalisation as the principal mode of changing one's belief state. This updating method fits the idea of the Lockean Thesis better than plain Bayesian conditionalisation, and it affords a flexible method for adding and withdrawing categorical beliefs. We show that it fails to satisfy the traditional principles of Inclusion and Preservation for belief revision and the principle of Recovery for belief withdrawals, as well as the Levi and Harper identities. We take this to be a problem for the latter principles rather than for the idea of coherent belief change.
\end{abstract}

Keywords Belief revision · Jeffrey conditionalization · Lockean Thesis · Coherence

Mathematics Subject Classification (2010) 68T30 · 68T37

\section{Introduction}

Belief can either be taken to be a binary yes/no notion (categorical belief, plain belief) or to come in degrees. These two conceptions of belief have turned out to be surprisingly hard to reconcile. It would be natural to think of the relation of categorical belief to degrees of belief as analogous to the relation between tall which is a categorical notion, and height that

John Cantwell

john.cantwell@abe.kth.se

\section{Hans Rott}

hans.rott@ur.de

1 KTH Stockholm, Stockholm, Sweden

2 SCAS Uppsala, Uppsala, Sweden

3 University of Regensburg, Regensburg, Germany 
comes in degrees (on some scale, say meters). Whether someone is to be considered tall depends on context and can, due to vagueness, be subject to borderline cases, but regardless of context, the question whether someone is tall or not depends on his or her height in a systematic fashion: for every context, if $x$ is tall and $y$ 's height is at least as great as $x$ 's height then $y$ is tall. The analogy would be that categorical belief in a similar fashion can be context dependent and allow for borderlines, but would still depend on degrees of belief ('credences') in a systematic fashion: for every context, if one categorically believes $A$ and one's degree of belief for $B$ is at least as great as one's degree of belief for $A$, then one (should) categorically believe $B$.

Indeed, such a relationship between categorical belief and degrees of belief is obtained if we adopt the:

Lockean Thesis (LT) A rational agent categorically believes $A$ if and only if the agent's degree of belief for $A$ is greater than (or equal to) $t$ (where $t$ is 'high', e.g. 0.9).

For if one's degree in $A$ exceeds $t$ and one's degree of belief in $B$ is greater than in $A$, then one's degree of belief in $B$ is also greater than $t$. A problem often cited for the Lockean thesis is the Lottery Paradox due to Kyburg [15]. It seems plausible that a rational agent can be confronted with a lottery in which the agent believes that some lottery ticket will win, but has a threshold $t$ for categorical belief such that for any lottery ticket $i$, the agent's degree of belief that $i$ will not win is greater than (or equal to) $t$. Together with LT, this would make the categorical beliefs of the agent jointly inconsistent, and so would be a violation of the principle of Consistency:

Consistency A perfectly rational agent does not have categorical beliefs that are jointly inconsistent.

Furthermore, the scenario depicted by the lottery paradox is also in conflict with:

Closure A perfectly rational agent believes any logical consequence of his or her beliefs. or slightly weaker:

Conjunctive Closure A perfectly rational agent believes the conjunction of any of his or her beliefs.

If one's beliefs are inconsistent, their conjunction is logically false, and, arguably, an agent, insofar as she is rational, does not commit herself to the truth of a logically false proposition.

If categorical belief is related to degrees of belief in a manner analogous to the way tall is related to height then either the threshold in the Lockean Thesis should be set to 1 (maximal degree of belief), or the principles of Consistency and Closure must be abandoned. There is a third strategy: let the threshold vary with context and be 'high enough' to avoid inconsistency, in a lottery with 1.000 tickets, for example, choose $t=0.9999$. A problem with such a strategy is that what comes to count as categorical belief will, except in special cases, have to be very close to certainty and so will only have limited informational value (though, as will be seen, by taking the resulting belief set as given and iterating this procedure, one will in important cases gain a non-trivial belief set).

Clearly, the reason for calling a possible violation of Consistency or Closure a 'paradox' does not derive from LT alone: there is nothing in LT that would suggest that categorical beliefs should be jointly consistent or closed under consequence. The norms of consistency and closure, or the appearance that there are such norms, must have a different source. As a 
set of highly probable propositions need be neither consistent nor closed, why would we ever think that categorical beliefs should abide by such norms?

One idea is that categorical belief and degrees of belief are not related in a manner analogous to the way tall is related to height, that they instead pick out different kinds of mental states with different kinds of functional roles. One can, for instance, think of degrees of belief as guiding action whereas categorical belief guides explicit judgments. On this model, degrees of belief or subjective probabilities are dispositive states that assign different weights to propositions, weights that form (or should form) the basis for making decisions in an open-ended range of possible decision situations where the stakes can vary freely. A categorical belief, on the other hand, would be a disposition to 'endorse' a proposition (judge it true), the kind of mental state that could form the basis for the assertion of a sentence expressing the proposition, or for what one takes for granted when reasoning about what act to perform. ${ }^{1}$ As what one is willing to assert and to take for granted may well depend on what is at stake in a given context, what counts as a categorical belief would on this account vary with context.

When degrees of belief and categorical beliefs are assigned very different explanatory roles in this way, there is no a priori reason to think that the two are related in the way stipulated by LT. We are under no general obligation to assert that a particular lottery ticket will not win even though it is highly probable that it won't, and so one may well deny that a perfectly rational agent is obliged to believe that a given lottery ticket will not win, irrespective of how likely it is that it won't win. Furthermore, it does not appear to be a norm of assertion that one assert only what one holds to be absolutely certain (e.g., analytical truths), so on the present way of interpreting categorical belief and degrees of belief, it would be implausible to insist that it is a norm that a rational agent should categorically believe only those propositions that have probability 1 .

This said, it would be strange if the two notions of belief as modelled here were completely unrelated, even though this relation cannot be spelled out in the manner LT. In this paper we want to explore two facets of how categorical belief and degrees of belief can be interconnected.

First, if degrees of belief and a contextually supplied threshold do not supply all the structure required for an analysis of categorical belief, what kind of additional structure would be required for such an analysis? In this paper we pursue a simple answer: it is sufficient that we have a partition of the space of the very many ways that things might be, a set of atomic 'possibilities' or indivisible 'possible worlds' that we can assign degrees of belief. Given a partition and a probability measure, one can delineate a space of coherent belief states and we will study how one can use thresholds to settle on a particular coherent belief set. Such a threshold can either be global (pertain to the probability of the closure of all of one's beliefs) or local (pertain to the probability of individual beliefs). It will be shown how a coherent belief set passing only a 'low' global threshold can be combined with a requirement that individual beliefs should pass a 'high' local threshold.

Second, probabilities can be updated. Orthodox Bayesianism allows for such updates by strict conditionalisation (updating a proposition with probability 1), while more liberal accounts allow for Jeffrey conditionalisation (updating a proposition with probability less than 1). It is the point of the Lockean Thesis to make room for categorical beliefs below maximal probability, and so it seems much more natural to couple LT with Jeffrey rather than with strict conditionalisation. Given that there is a link between confidence in

\footnotetext{
${ }^{1}$ For arguments in favour of the parallel between belief and assertion, see Adler [1] and Douven [8].
} 
categorical belief on the one hand and degrees of belief on the other, one would expect an update in probabilities to have effects on what one categorically believes. Conversely, given that one changes one's categorical beliefs (by expanding, revising or contracting belief), one would expect that one's underlying degrees of belief should change as well. In this paper we want to study how one can expand, revise and contract one's categorical beliefs by means of Jeffrey conditionalising one's credences in such a way as to maintain coherence among categorical beliefs.

\section{Preliminaries}

\subsection{Belief sets, probability measures and belief states}

Everything we are going to say in this paper is restricted to a finite setting. Let $U$ be a finite set of 'possibilities' or '(possible) worlds', i.e., our partition of the ways things might be. We will in general treat $U$ as given in the following. Any subset $A$ of $U$ counts as a proposition; its complement in $U$ will be denoted by $\bar{A}$. A proposition is consistent if it is non-empty, a set of propositions is consistent if their intersection is non-empty. A (possible) belief set is a subset Bel of $U$ (and so a proposition). The set of beliefs induced by, or the set of propositions believed in, a belief set Bel, in symbols $\mathcal{B}(\mathrm{Bel})$, is the set $\{A \subseteq U: B e l \subseteq A\}$. The belief set $\mathrm{Bel}$ is also said to be the core of $\mathcal{B}(\mathrm{Bel})$. Note that $\mathcal{B}(\mathrm{Bel})$ will be closed under unions and intersections (henceforth, simply closed). Note also that if $\mathrm{Bel} \subseteq \mathrm{Bel}^{\prime}$, then $\mathcal{B}\left(\mathrm{Bel}^{\prime}\right) \subseteq \mathcal{B}(\mathrm{Bel})$ - the smaller the belief set the larger the set of beliefs. A set of basic beliefs is a set $\mathcal{B}_{\text {basic }}$ of propositions. The belief set induced by a set of basic beliefs is the intersection: $\bigcap \mathcal{B}_{\text {basic }}$. One can now understand $\mathcal{B}\left(\bigcap \mathcal{B}_{\text {basic }}\right)$ as the set of beliefs that follow from the basic beliefs $\mathcal{B}_{\text {basic }}$. These are the 'derivable beliefs' or the propositions the agent is 'committed to' if her basic beliefs are $\mathcal{B}_{\text {basic }}$. If $\mathcal{B}\left(\cap \mathcal{B}_{\text {basic }}\right)=\mathcal{B}_{\text {basic }}$, then the basic beliefs are said to be closed.

A probability measure $\operatorname{Pr}$ over $U$ is a real-valued function satisfying, for any $A, B \subseteq U$, (i) $0 \leq \operatorname{Pr}(A) \leq 1$, (ii) $\operatorname{Pr}(U)=1$, and (iii) $\operatorname{Pr}(A \cup B)=\operatorname{Pr}(A)+\operatorname{Pr}(B)$ if $A$ and $B$ are disjoint. A probability measure is regular if (iv) If $\operatorname{Pr}(A)=1$, then $A=U$. We abbreviate ' $\operatorname{Pr}(\{u\})$ ' by ' $\operatorname{Pr}(u)$ '. Conditional probabilities $\operatorname{Pr}(A \mid B)$ are defined as usual as $\operatorname{Pr}(A \cap B) / \operatorname{Pr}(B)$, whenever $\operatorname{Pr}(B)>0$. A measure is non-uniform if there are at least two worlds with different probabilities, and it is positively non-uniform if there are at least two worlds with different positive probabilities.

Since $U$ is supposed to be finite, we may use representations of $\operatorname{Pr}$ of the format depicted in Fig. 1. The boxes are supposed to contain $k_{i}$ (possible) worlds with probability values

Fig. 1 Layer representation of a finite probability function

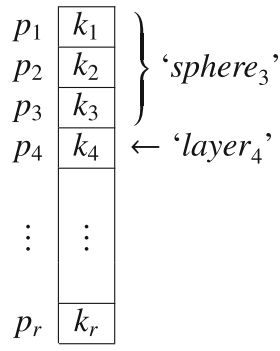


$p_{i}$, where the probability values are listed in decreasing order, i.e., $p_{1}>p_{2}>p_{3}>p_{4}>$ $\cdots>p_{r} \geq 0$ (we do not exclude $p_{r}=0$ ). Obviously, any $k_{i}$ is a non-negative integer, and we assume as a convention that our representation rules that for no $i$, we have $k_{i}=0$ (no empty layers). The set of $k_{i}$ worlds with probability $p_{i}$ is called layer $_{i}$, and the set of $\sum_{j=1}^{i} k_{j}$ worlds residing in the $i$ upper layers is called sphere ${ }_{i}$.

Our central notion is that of a belief state. A belief state is a pair $(\mathrm{Pr}, \mathrm{Bel})$ where $\mathrm{Pr}$ is a probability measure and $\mathrm{Bel}$ is a belief set. We consider the belief set and the probability function as two components of the agent's doxastic state.

\subsection{Coherence}

The model we are going to study is fundamentally based on the idea that belief states be coherent in the following sense.

Definition 1 A belief state $(\mathrm{Pr}, \mathrm{Bel})$ is said to be coherent if $\mathrm{Bel}$ is consistent and for any worlds $u$ and $v$ in $U$, if $u$ is in Bel and $\operatorname{Pr}(u) \leq \operatorname{Pr}(v)$, then $v$ is in Bel, too.

Given a fixed probability measure $\mathrm{Pr}$, a belief set $\mathrm{Bel}$ will be said to be coherent with respect to $\mathrm{Pr}$, or if no confusion can arise, simply coherent, if $(\mathrm{Pr}, \mathrm{Bel})$ is coherent. This is an idea of static coherence, and its motivation is based on a principle of sufficient reason: ${ }^{2}$ if a world $u$ has been excluded and $v$ is not more probable than $u$, then $v$ should be excluded as well. Further motivation for this kind of coherence will be given in Section 3.

Observation 1 If $(\mathrm{Pr}, \mathrm{Bel})$ and $\left(\mathrm{Pr}, \mathrm{Bel}^{\prime}\right)$ are two coherent belief states then either $\mathrm{Bel} \subseteq$ $B e l^{\prime}$ or $B e l^{\prime} \subseteq B e l$.

The coherent belief states relative to a probability measure are thus linearly ordered in terms of strength. It is due to this nestedness property that we sometimes call coherent belief sets 'spheres' (after the system of spheres representations of Lewis [22] and Grove [9]). A belief set $\mathrm{Bel}$ is regular if for no world $u \in \mathrm{Bel}, \operatorname{Pr}(u)=0$. Trivially, there can be no irregular belief sets if the underlying measure $\mathrm{Pr}$ is itself regular, and if $\mathrm{Pr}$ is not regular, then the only coherent irregular belief set is $U$. Given an irregular measure, there will be a proposition $A \neq U$ such that $\operatorname{Pr}(A)=1$. In a coherent irregular belief set $\mathrm{Bel}$ one could thus have $A \notin \mathcal{B}(B e l)$ even though $\operatorname{Pr}(A)=1$ (simply let $B e l=U$ ). This is strange, and for this reason we will mostly consider regular belief sets. A belief set Bel is informative if $\emptyset \neq B e l \neq U$. A belief set Bel is $P r$-informative (with respect to a probability measure $P r$ ) if $0 \neq \operatorname{Pr}(\mathrm{Bel}) \neq 1$. Clearly, all $\operatorname{Pr}$-informative belief sets are informative, but in general not vice versa.

\subsection{Global and local thresholds}

The Lockean thesis determines a belief set when a probability measure and a threshold are given. We propose the following alternative to the Lockean thesis:

\footnotetext{
${ }^{2}$ Cf. Rott and Pagnucco [30] for the application of such a principle in a qualitative setting for the dynamics of belief.
} 
Definition 2 (Coherentist Lockean thesis) The belief set determined by a probability measure $\operatorname{Pr}$ over a set of worlds $U$ and a global threshold $t$, in symbols $\mathrm{Bel}_{t}(\mathrm{Pr})$, is the smallest belief set $\mathrm{Bel}$ such that

(i) Bel is coherent with respect to $\operatorname{Pr}$, and

(ii) $\operatorname{Pr}(\mathrm{Bel}) \geq t$.

Here the global threshold is just a real number $t$ in the interval $(0,1]$. Observation 1 guarantees the existence of a smallest belief set satisfying (i) and (ii). We say that Pr accepts a proposition $A$ at threshold $t$ if $\mathrm{Bel}_{t}(\mathrm{Pr}) \subseteq A$.

In this way, a probability measure $\operatorname{Pr}$ over $U$ and a threshold $t$ will jointly determine a coherent belief state. Since conversely a coherent belief set $\mathrm{Bel}$ is determined by setting the threshold $t$ to $\operatorname{Pr}(\mathrm{Bel})$, every coherent belief state $(\mathrm{Pr}, \mathrm{Bel})$ can equivalently be represented as a pair $(P r, t)$. Whenever we take the threshold to be given by the context and we insist that belief states need to be coherent, a belief state can be represented through a probability measure alone.

Given a threshold $t$ between 0 and 1 (excluding 0 ), a probability function $\operatorname{Pr}$ represented like in Fig. 1 determines the belief set $B_{t}(P r)$ in the following way. Define $i(t)$ to be the index $i$ such that $\sum_{j=1}^{i} n_{j} \cdot p_{j} \geq t$, but $\sum_{j=1}^{i-1} n_{j} \cdot p_{j}<t$. Then $B_{t}(P r)=\{u \in U$ : $\left.\operatorname{Pr}(u) \geq p_{i(t)}\right\}$. This is the proposition that equals the minimal minimal upper sphere of $\operatorname{Pr}$ the total probability of which exceeds the threshold $t$.

Figure 2 presents an example where $U$ has 18 worlds, $P r$ assumes six different values and $t$ is set to 0.75 . The upper four layers ('sphere 4 ') are needed to reach the threshold value, that is, $i(t)=4$.

Global thresholds relate to belief sets. If one takes a belief set to be derived from a set of basic beliefs $\mathcal{B}_{\text {basic }}$ then the global threshold does not relate to the individual members of $\mathcal{B}_{\text {basic }}$, but to their intersection. The global threshold thus sets a lower limit for how improbable the intersection of a set of basic beliefs may be.

A threshold that applies to one or more individual members of a set of basic beliefs is called a local threshold. Local thresholds will always be assumed to be in the interval $(0.5,1]$ (contrast this to the interval for global thresholds).

Given a belief state $(\mathrm{Pr}, \mathrm{Bel})$, a set of basic beliefs $\mathcal{B}_{\text {basic }}$ satisfies a local threshold $t$ if $\operatorname{Pr}(A) \geq t$ for all $A \in \mathcal{B}_{\text {basic }}$. A set of basic beliefs $\mathcal{B}_{\text {basic }}$ is determined by a local threshold $t$ iff $\mathcal{B}_{\text {basic }}=\{A \subseteq U: \operatorname{Pr}(A) \geq t\}$. So if a set of basic beliefs is determined by a local threshold $t$ it will satisfy the Lockean Thesis (LT).

A belief set Bel is locally threshold based (relative to $P r$ ) if there is some threshold $t$ such that

$$
\text { Bel }=\bigcap\{A: \operatorname{Pr}(A) \geq t\} .
$$

Fig. 2 A six-layered example, with $t=0.75$. Here and in the following figures, belief sets are indicated by shaded layers

\begin{tabular}{l|l|l}
\cline { 2 - 2 }$p_{i}$ & $n_{i}$ & $\operatorname{Pr}\left(\right.$ sphere $\left._{i}\right)$ \\
\cline { 2 - 2 } 0.20 & 2 & 0.4 \\
0.12 & 1 & 0.52 \\
0.07 & 2 & 0.66 \\
0.05 & 4 & 0.86 \\
0.02 & 5 & 0.96 \\
0.01 & 4 & 1
\end{tabular}


So a set of basic beliefs satisfying the Lockean Thesis will induce a locally threshold based belief set $B e l$. We know from the lottery and preface paradoxes that $\bigcap\{A: \operatorname{Pr}(A) \geq t\}$ may well be empty, but this of course does not apply to all situations and all thresholds.

Turning to Fig. 2, the belief set we get with global threshold $t_{G}=0.75$ will be locally threshold based relative to any local threshold $t_{L}$ such that $0.95<t_{L} \leq 0.98$. The lower bound comes from the fact that every world in all the four upper layers must be included in every proposition exceeding the threshold (if $t_{L} \leq 0.95$ the proposition $U-\{u\}$, where $u$ is any world belonging to the fourth layer would exceed the threshold, as $\operatorname{Pr}(u)=0.05$ ). The upper bound comes from the constraint that no particular single world in the lower two layers must be in a proposition in order to reach the threshold (if $t_{L}>0.98$ then every proposition exceeding the threshold needs to contain all the worlds in the fifth layer as these have probability 0.02 ).

Given a (consistent) locally threshold based belief set $\mathrm{Bel}$ on $\mathrm{Pr}$ one can form a new probability measure $P r_{B e l}$ by conditionalising $\operatorname{Pr}$ on $B e l$ (so $\operatorname{Pr} B e l()=.\operatorname{Pr}(. \mid B e l)$ ). This new probability measure will generate a new set of locally threshold based belief sets and each of these can in turn be used to generate yet a new probability measure by conditionalisation, and so on. In general: a belief set Bel is iteratedly threshold based (relative to Pr) if there are belief sets $B e l_{0}, \ldots, B e l_{n}$ such that:

1. $B e l_{0}$ is locally threshold based relative to $\operatorname{Pr}$.

2. For each $i, 0<i<n: B e l_{i+1}$ is locally threshold based relative to $\operatorname{Pr}_{B e l_{i}}$.

3. Bel is locally threshold based relative to $\operatorname{Pr}_{B e l_{n}}$.

Again turning to Fig. 2, let $B e l_{0}$ be locally threshold based relative to $P r$ with a local threshold $t_{L}$ in the interval $0.95<t_{L} \leq 0.98$. Bel $l_{0}$ thus contains the worlds in the four top layers. Let $B e l_{1}$ be locally threshold based relative to $P r_{B e l_{0}}$ with a local threshold $t_{L}$ in the interval $0.93 \leq t_{L} \leq 0.94$. $B e l_{1}$ will contain the worlds of the upper three layers. And so on: letting $\mathrm{Bel}_{2}$ be the upper two layers it is locally threshold based relative to $\mathrm{Pr}_{B e l_{1}}$, and letting $\mathrm{Bel}_{3}$ be the upper first layer it is locally threshold based relative to $\mathrm{Pr}_{\mathrm{Bel}_{2}}$ (indeed, as will be shown in the next section, this is a general property of coherent sets).

\section{Motivating coherence}

In this section we try to further motivate the idea that belief states should be coherent, i.e. the constraint that for all $u, v \in U$, if $u \in B e l$ and $\operatorname{Pr}(u) \leq \operatorname{Pr}(v)$ then $v \in$ Bel. $^{3}$

First note some properties of coherent belief sets.

\section{Observation 2}

(i) Every consistent locally threshold based belief set is coherent.

\footnotetext{
${ }^{3}$ Before motivating the coherence constraint one should perhaps motivate why we at all call it 'coherence'. While we are not entirely happy with using a term with so many uses and connotations we think the nomenclature can to some extent be defended. First, unlike consistency, it is a non-logical property and, like consistency, it is a property of whole belief sets rather than individual beliefs. Second, as shown by Cantwell [4], the formal property of coherence corresponds to (or is, at least, a formal interpretation of) the pre-formal notion of coherence championed by Keith Lehrer [16]; so at the very least the use has a historical precedent.A rather large variety of 'integration assumptions' describing ways in which belief states $(\mathrm{Pr}, \mathrm{Bel})$ may be 'coherent' is discussed by Leitgeb [18, Chapter 1].
} 
(ii) For every non-uniform probability measure on $U$, there is at least one informative locally threshold based belief set Bel.

(iii) For every positively non-uniform probability measure on $U$, there is at least one $\mathrm{Pr}$ informative locally threshold based belief set $\mathrm{Bel}$.

The proofs of our observations are collected in an Appendix at the end of the paper.

Now we know that for any (positively) non-uniform probability measure there is a nontrivial local threshold $t(<1)$ such that the set of propositions with probability greater than $t$ constitute a coherent (and so consistent) $P r$-informative set of basic beliefs $\mathcal{B}_{\text {basic }}$. That is, for any (positively) non-uniform probability measure there is a non-trivial local threshold relative to which the Lockean Thesis is 'well-behaved' in the sense that the resulting set of basic beliefs form a coherent (and so consistent) base. One can show some further properties:

\section{Observation 3}

(i) There is a strongest consistent locally threshold based belief set.

(ii) Every coherent belief set that contains the strongest consistent locally threshold based belief set, is itself a locally threshold based belief set.

All locally threshold based belief sets are coherent. However, the converse need not hold. So even if one takes a belief set to be generated by a set of basic beliefs, high probability (short of probability 1) is not in general a sufficient criterion for belief. It won't in general be the case that a coherent belief set is induced by a set of basic beliefs determined by a local threshold. However, one can show the following:

Observation 4 For any coherent belief set $\mathrm{Bel}$, there is a set of propositions $\mathcal{B}_{\text {basic }}$ satisfying a local threshold $t>0.5$ (so $\operatorname{Pr}(A) \geq t$ for all $A \in \mathcal{B}_{\text {basic }}$ ) such that $\mathcal{B}_{\text {basic }}$ induces $\mathrm{Bel}$.

So even if a coherent belief set $\mathrm{Bel}$ is determined by some global threshold $t_{G}<0.5$, it can always be seen as being induced from a set of basic beliefs exceeding a local threshold $t_{L}>0.5$. In addition, for any local threshold $t_{L}>0.5$ there will be a strongest coherent belief set $\mathrm{Bel}$ such that some set $\mathcal{B}_{\text {basic }}$ of propositions exceeding the threshold induces Bel. As far as individual basic beliefs are concerned, one can insist that high probability is a necessary condition for rational acceptance.

Not every coherent belief set is locally threshold based. However we have:

Observation 5 Every coherent belief set is iteratedly threshold based.

Indeed we also have its converse:

Observation 6 Every consistent iteratedly threshold based belief set is coherent.

So, the coherent belief sets are precisely the (consistent) iteratedly threshold based belief sets. This opens up for an alternative way of defining coherence: one can define it in terms of the property of being iteratedly threshold based. As the latter notion makes sense for any probability measure on any space (a space that need not be finite and that need not have atoms or 'possible worlds'), this could be a way of getting rid of the partition-dependency of the present framework. However, this avenue will not be explored further here. 
The following picture emerges: one's beliefs are the propositions one takes for granted; at a first level these would have to be highly probable to yield a coherent belief state; however, once one takes these propositions for granted, new propositions will be able to pass the local threshold, and so these can be taken for granted, and so on until one reaches a point corresponding to one's global threshold. The result is a coherent belief set. On this picture the global threshold imposes a probabilistic lower bound for the belief set as a whole, thus putting a limit to how far one can iterate the process of forming a locally threshold based belief set and conditionalising on the result.

\section{Changing belief states}

A probability measure is a more fine-grained kind of representation than a proposition. We interpret the probability measure as the principal component of an agent's doxastic state $(\mathrm{Pr}, \mathrm{Bel})$. Changes of belief states are effected by changes of the probabilities, which then combine with the currently effective threshold $t$ to produce the belief set Bel. We will consider only changes of belief states that do not involve a change in the threshold value $t$. A change of $t$-and a change of Bel that is due just to this change of $t$-should perhaps be counted as a merely pragmatic (rather than genuinely doxastic) change. It is hard to imagine that a change of the threshold might be a matter of receiving new evidence.

\subsection{Changing probability measures by Jeffrey conditionalisation}

Ordinary Bayesian conditionalisation commits the agent to endorse any new information with maximal certainty. But the Lockean Thesis, and equally the Coherentist Lockean Thesis, insist that beliefs do not require maximal certainty. So ordinary conditionalisation seems too strict. Richard Jeffrey $[12,13]$ introduced a method of accepting evidence to a specific degree $\alpha$ between 0 and 1 . When $\operatorname{Pr}$ is a probability measure, $\alpha \in[0,1]$ and $A$ is a proposition such that $0<\operatorname{Pr}(A)<1$, let us define:

\section{Jeffrey conditionalisation $\quad \operatorname{Pr}_{A}^{\alpha}(B)=\alpha \cdot \operatorname{Pr}(B \mid A)+(1-\alpha) \cdot \operatorname{Pr}(B \mid \bar{A})$.}

Assuming in the following that the probability of $A$ is neither 0 nor 1 , we suggest that belief revision by $A$ should go by Jeffrey conditionalisation. More precisely, to rationally revise one's belief state by $A$ is to make a rational update of one's credences in such a way that one comes to believe $A$. Formally:

Definition 3 Suppose that an agent is in the belief state $(\operatorname{Pr}, t)$ and that $0<\operatorname{Pr}(A)<1$. Then the belief state $\left(\operatorname{Pr}^{\prime}, t\right)$ is a result of rationally revising by $A$ iff there is an $\alpha \geq \operatorname{Pr}(A)$ such that

(i) $\operatorname{Pr}^{\prime}=\operatorname{Pr}_{A}^{\alpha}$, and

(ii) $\operatorname{Bel}_{t}\left(\mathrm{Pr}^{\prime}\right) \subseteq A$.

To rationally update one's belief state by $A$ (given an unchanging threshold $t$ ) thus is to Jeffrey conditionalise one's probability measure $\operatorname{Pr}$ on $A$ in such a way that one comes to believe that $A$. We base our considerations in the following on the assumption that every rational way of updating by $A$ is the result of a Jeffrey conditionalisation. However, due to the constraints that the result should be a coherent belief state in which one believes that $A$ (constraint (ii) of Definition 3), the converse will not in general hold: there can be Jeffrey 
conditionalisations with $A$ that do not constitute a way of rationally revising by $A$. This usually happens when the Jeffrey parameter $\alpha$ is chosen too low.

Our way of combining the ideas of coherence and Jeffrey conditionalisation can be applied in almost exactly the same way in order to define an operation of withdrawing $A$ or 'downdating' by $A .{ }^{4}$ A withdrawal produces a belief set $\operatorname{Bel}_{t}\left(\operatorname{Pr}_{A}^{\alpha}\right)$ that does not entail $A$.

Definition 4 Suppose that an agent is in the belief state $(P r, t)$. Then the belief state $\left(P r^{\prime}, t\right)$ is a result of rationally withdrawing $A$ iff there is an $\alpha \leq \operatorname{Pr}(A)$ such that

(i) $\operatorname{Pr}^{\prime}=\operatorname{Pr}_{A}^{\alpha}$, and

(ii) $\operatorname{Bel}_{t}\left(\operatorname{Pr}^{\prime}\right) \nsubseteq A$.

We now have an idea of how probability measures and belief sets are being changed, provided that the global threshold $t$ remains unaltered. Taking $t$ to be pragmatically fixed by the context, the corresponding update of a belief state $(\mathrm{Pr}, \mathrm{Bel})$ or $(\mathrm{Pr}, t)$ by a proposition $A$ flows naturally from the update of the probability measure: it is $\left(\operatorname{Bel}_{t}\left(\operatorname{Pr}_{A}^{\alpha}\right), \operatorname{Pr}_{A}^{\alpha}\right)$ or $\left(t, \operatorname{Pr}_{A}^{\alpha}\right)$, respectively, with $\alpha$ determined by $t$ in ways we are now going to explore.

As our model combines ideas that are attributable to coherentism, Locke and Jeffrey, we call it the Coherentist Locke-Jeffrey model of belief change. There is yet another important element that we will soon add to it, namely the idea of minimal change, but the name is long enough. We don't want to burden it any further.

\subsection{Global and local thresholds: the relationship between $t$ and $\alpha$}

It is important to remember that the global threshold $t$ applies to the belief set as a whole and not to the posterior probability $\alpha$ of the proposition $A$ that is to be believed or 'accepted'. In a successful revision it will always be the case that $\alpha \geq t$. But the goal of coherence does not come for free. Some propositions do not require much evidence to fit smoothly into one's set of beliefs, other propositions would require far more solid evidence as accommodating them would require substantial revisions of one's existing beliefs in order to maintain coherence. So typically - apart from special cases - the only way of learning $A$ will be by Jeffreyconditionalising on $A$ with some parameter $\alpha$ that is strictly larger than $t$ (examples will be given in Section 5).

The existence of a suitable $\alpha$ is guaranteed regardless of the threshold $t$ (assuming that $\operatorname{Pr}(A)>0)$ : we have $\operatorname{Bel}_{t}\left(\operatorname{Pr}_{A}^{1}\right) \subseteq A$, so $\alpha=1$ will always accept $A$. Uniqueness, however, is not guaranteed: except for special cases there will be a continuum of possible ways of revising by $A$.

We have placed very weak restrictions on the global threshold $t$ that governs the belief set as a whole. All that is required is that it is in the interval $(0,1]$. This means that the probability for one's belief set can be low, below 0.5 , indeed lower still. This is in contrast to the tradition that holds that high probability is required for belief..$^{5}$ Indeed in many contexts, contexts where one is modelling only a few limited aspects of the world, a high global threshold for the belief set seems natural. But when one widens the perspective and considers an agent faced with a richer set of aspects of the world there is a way of making sense of low-probability and very low-probability global thresholds. For the belief set is the logical closure of one's individual beliefs. Is it that strange to allow that a rational agent-even a

\footnotetext{
${ }^{4}$ The felicitous term 'downdating' is probably due to van Benthem [32].

${ }^{5}$ For a dissenting voice, however, see Kaplan [14].
} 
logically omniscient agent—can hold it to be unlikely that everything that logically follows from his or her beliefs is true?

The paradox of the preface is a case in point. The disclaimer that can come when presenting a sizeable collection of one's beliefs (as can be done in book form), to the effect that it is quite unlikely that all the claims are true,${ }^{6}$ reflects a view of belief where the threshold for the conjunction of all one's relevant beliefs can be low. Whether the conjunction of all the claims an author makes in a book is to be considered a full fledged belief in its own right is debatable (closure under conjunction), but clearly there is a sense in which the author is committed to its truth. For no matter that the author finds the conjunction unlikely: if the conjunction is false this is only because at least one of the particular claims is false, and in making each particular claim, the author commits to that claim. The constraint that the probability of one's combined beliefs should exceed 0 and so should be logically consistent then reflects the constraint that whether, say, $A$ should count as one of one's beliefs should at least in a minimal way cohere with one's other beliefs.

The present framework at least allows that the sum total of one's beliefs have a low probability of being true (although, recall Observation 4, one can still insist that basic beliefs have a high probability). However, even if one takes the potential for low probability (inferred) beliefs to be a good feature (as it, for instance, helps us understand the paradox of the preface), one might hold that this only applies to one's existing beliefs. One might still insist that any new belief that is added should have a high probability. To incorporate this idea we must distinguish the global threshold (which applies to the belief set as a whole) and the local threshold which applies to the acquisition of individual beliefs. ${ }^{7}$

\section{Constructing changes of belief states}

Like many authors, we take rationality to further include the idea of minimal change or doxastic conservatism. Jeffrey conditionalisation can in itself be interpreted as encoding this idea (see Diaconis and Zabell [6] and Dietrich, List and Bradley [7]). But there is a further interpretation of minimal change that may be taken to act as a norm for rational agents.

Revision by $A$ raises the probability of $A$, while the withdrawal of $A$ lowers its probability. Minimal change requires that one take a minimal $\alpha$ that achieves the task of accepting $A$ in accordance with Definition 3, and the maximal $\alpha$ that achieves the task of unaccepting $A$ in accordance with Definition 4. (A revision leaves $A$ 's probability unchanged if $A$ is already believed, and similarly, a withdrawal does nothing if $A$ is not believed in the current belief state.) In Jeffrey's work $\alpha$ reflects the quality of the evidence (cf. his famous 'observation by candlelight'), while we aim at finding just the minimal $\alpha-$ or, if a minimal solution is not available, a very low $\alpha$-that makes $A$ coherently acceptable when the (global) threshold $t$ is in place (where $t$ is not an epistemic parameter, but a pragmatic one

\footnotetext{
${ }^{6}$ In this version of the paradox of the preface one concedes that it is likely that some claim (in the book) is false. Our model cannot deal with the stronger disclaimer that some claim in the book must be false. However, we are not convinced that one ever can be warranted in being sure that one has a false belief.

${ }^{7}$ This strikes an old familiar pragmatic chord according to which the justification of one's present beliefs is of less concern than the justification of when and how to revise one's beliefs: one can view the local threshold as the threshold for letting new beliefs in, and the global threshold as the threshold of how unlikely one is willing one's totality of beliefs to become. The latter may be low even when the former is high. The point has been especially forcefully made by Isaac Levi who credits the idea to Charles Sanders Peirce: 'Peirce ... was a fan of the principle of doxastic inertia according to which there is no need to justify current beliefs (i.e., doxastic commitments) but only changes in belief (doxastic commitments)' [21, p. 179].
} 
given by the context). The question we are asking is thus, given one's global threshold: how low a quality of evidence in favour of $A$ would suffice for accepting $A$ ? Or, in the case of withdrawal: how low a quality of evidence speaking against $A$ would suffice for giving up $A$ ? In the coming sections we study some properties of such minimal changes.

If $A$ has non-zero probability there will be some Jeffrey parameter $\alpha$ such that the Jeffreyconditionalisation $\operatorname{Pr}_{A}^{\alpha}$ yields a belief set in which $A$ is accepted. As noted, there need not in all cases and for all thresholds be a minimal parameter that accepts $A$, but a Jeffrey parameter $\alpha$ will be said to be minimising for revision with $A$ if for all $\alpha^{\prime} \leq \alpha$ such that $\alpha^{\prime}$ is sufficient to accept $A$ :

$$
\operatorname{Bel}_{t}\left(\operatorname{Pr}_{A}^{\alpha}\right)=\operatorname{Bel}_{t}\left(\operatorname{Pr}_{A}^{\alpha^{\prime}}\right) .
$$

That is, a Jeffrey parameter is revision minimising for $A$ if no smaller parameter that is sufficient for accepting $A$ will yield a different belief set. If there is some way of revising with $A$, there is a minimising way of revising with $A$.

Similarly, when $A$ has a probability less than 1 and greater than 0 , there will be a parameter $\alpha$ that leads to $A$ being withdrawn ('unaccepted') without $\bar{A}$ being accepted. One does this by decreasing the probability of $A$ so here larger values for $\alpha$ will be more conservative than lower values. Again there need not always be a maximal such value, in which case there is no smallest change that ensures that $A$ is withdrawn. A Jeffrey parameter $\alpha$ will be said to be minimising for withdrawal of $A$ if for all $\alpha^{\prime} \geq \alpha$ such that $\alpha^{\prime}$ withdraws $A$ :

$$
\operatorname{Bel}_{t}\left(\operatorname{Pr}_{A}^{\alpha}\right)=\operatorname{Bel}_{t}\left(\operatorname{Pr}_{A}^{\alpha^{\prime}}\right) .
$$

That is, a Jeffrey parameter is minimising for withdrawal if no greater (more conservative) parameter will yield a different belief set (with the idea that what is being minimised is the effect of the withdrawal on the belief set).

In the remainder of this section this is spelled out with more formal rigour. We will give two recommendations what to do in the absence of minimal revision solutions in Section 5.2 and three recommendations for what to do in in the absence of maximal withdrawal solutions in Section 5.4.

\subsection{Finding the right Jeffrey parameters for changes of belief sets}

Since we are interested in a Jeffrey conditionalisation of a probability function $\operatorname{Pr}$ by $A$ and we are working in a finite setting, we use the representation of $\mathrm{Pr}$ as shown in Fig. 3. It is just a refinement of the representation in Fig. 1. The boxes are again supposed to contain (possible) worlds with probability values $p_{i}$, where the probability values are listed in decreasing order. Here $n_{i}$ denotes the number of worlds in $A$ with probability $p_{i}$, and $m_{i}$ the number of worlds in $\bar{A}$ with probability $p_{i}$. Obviously, $n_{i}$ and $m_{i}$ are non-negative integers, and one of them may well be 0 . However, our representation presupposes that we have $n_{i}+m_{i}>0$ for every $i$ (again, no empty layers). Clearly, $\operatorname{Pr}(A)=\sum_{i=1}^{r} n_{i} \cdot p_{i}$, and $\operatorname{Pr}(\bar{A})=\sum_{i=1}^{r} m_{i} \cdot p_{i}$.

Given a probability measure $P r$, we call the set of $n_{i} A$-worlds with probability $p_{i} A$ -

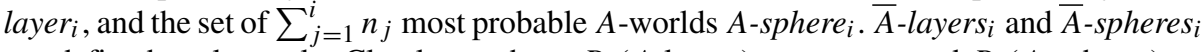
are defined analogously. Clearly, we have $\operatorname{Pr}\left(A\right.$-layer $\left._{i}\right)=n_{i} \cdot p_{i}$ and $\operatorname{Pr}\left(A\right.$-sphere $\left._{i}\right)=$ $\sum_{j=1}^{i}\left(n_{j} \cdot p_{j}\right)$.

Let $i(\bar{A})$ be the index of the first non-empty $\bar{A}$-layer, i.e., the lowest $i$ such that $m_{i} \neq 0$ (we suppose that there is such an $i$ with $p_{i} \neq 0$, i.e., that $\operatorname{Pr}(A)<1$ ).

The upper A-sphere of $P r$, in symbols $U p p A(P r)$, is defined to be the set of $A$-worlds that are more probable than any $\bar{A}$-world, that is, $\{u \in A: \operatorname{Pr}(u)>\operatorname{Pr}(v)$ for all $v \in \bar{A}\}$, or 
Fig. 3 A finite probability function, with $A$-worlds and $\bar{A}$-worlds separated

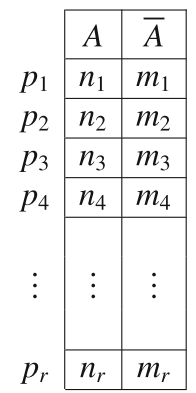

equivalently, the set of the $n_{1}+\cdots+n_{i(\bar{A})-1}$ most probable $A$-worlds. We put $\operatorname{Upp} A(\operatorname{Pr})=$ $\emptyset$ if $i(\bar{A})=1$. The upper $A$-sphere is the largest coherent set consisting exclusively of $A$-worlds.

Given a global threshold $t$, the probability function $\operatorname{Pr}$ represented as in Fig. 3 determines the belief set $\operatorname{Bel}_{t}(\mathrm{Pr})$ as the minimal set of upper layers the total probability of which exceeds the threshold $t$. Let $i(t)$ be the index for which $\sum_{i=1}^{i(t)}\left(n_{i}+m_{i}\right) \cdot p_{i} \geq t$, but $\sum_{i=1}^{i(t)-1}\left(n_{i}+m_{i}\right) \cdot p_{i}<t$. Then $\operatorname{Bel}_{t}(\operatorname{Pr})=\left\{u \in U: \operatorname{Pr}(u) \geq p_{i(t)}\right\}$. Now we can immediately make the simple

Observation 7 Given a probability function $\operatorname{Pr}$, the following conditions are equivalent:

(i) $\operatorname{Bel}_{t}(\operatorname{Pr}) \subseteq A$;

(ii) $\operatorname{Pr}(\operatorname{Upp} A(\operatorname{Pr})) \geq t$;

(iii) $i(t)<i(\bar{A})$.

If $\mathrm{Bel}_{t}(\mathrm{Pr}) \subseteq A$, then $\mathrm{Bel}_{t}(\mathrm{Pr}) \subseteq U p p A(P r)$, but the reverse inclusion $U p p A(P r) \subseteq$ $B_{e} l_{t}(P r)$ does not generally hold. The reach of $A$ may extend further than the belief set defined by $\operatorname{Pr}$ and $t$.

Jeffrey conditionalisation can achieve a qualitative belief revision or withdrawal by blowing up or shrinking the upper $A$-sphere just to the extent that is necessary for $A$ ending up being accepted or unaccepted, respectively. After a Jeffrey conditionalisation by $A$ with certainty parameter $\alpha$, all the worlds in $A$ with probability $p_{i}$ get a new probability $\frac{\alpha}{\operatorname{Pr}(A)} \cdot p_{i}$, while the worlds in $\bar{A}$ with probability $p_{i}$ get a new probability $\frac{1-\alpha}{\operatorname{Pr}(\bar{A})} \cdot p_{i}$ (see Fig. 4).

\subsection{Belief revision}

Given a probability function $\mathrm{Pr}$ and a (contextually determined) global threshold $t$, the operation of rationally revising by $A$ can be determined in the following way. For any $i$, define $\alpha_{i}$ as the number such that after Jeffrey conditionalisation $\operatorname{Pr}_{A}^{\alpha_{i}}$, the $A$-worlds at layer $i$ become exactly as probable as the most probable $\bar{A}$-worlds (we apply this definition also if $\left.n_{i}=0\right)$. That is, $\alpha_{i}$ is determined such that the following equality holds:

$$
\frac{\alpha_{i}}{\operatorname{Pr}(A)} \cdot p_{i}=\frac{1-\alpha_{i}}{\operatorname{Pr}(\bar{A})} \cdot p_{i(\bar{A})} .
$$

This is equivalent to

$$
\alpha_{i}=\frac{p_{i(\bar{A})} \cdot \operatorname{Pr}(A)}{p_{i} \cdot \operatorname{Pr}(\bar{A})+p_{i(\bar{A})} \cdot \operatorname{Pr}(A)} .
$$


Fig. 4 The result of Jeffreyconditionalising the probability function given in Fig. 3 by $A$ with certainty parameter $\alpha$ (creating up to $2 \cdot r$ new probability layers, not yet rearranged according to their probability values)

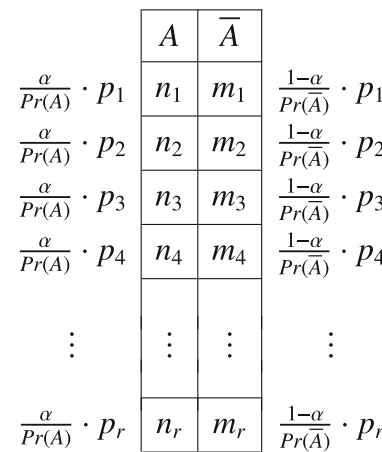

Equation (2) is defined if the denominator is positive, that is, if $0<\operatorname{Pr}(A)<1$. Note that $\alpha_{1}<\cdots<\alpha_{r}$. If $\alpha$ is set to $\alpha_{i}$, then the upper $A$-sphere of $\operatorname{Pr}_{A}^{\alpha}$ is $A$-sphere -si-1 $_{\text {(and the }}$ empty set for $i=1$ ). After the Jeffrey conditionalisation $\operatorname{Pr}_{A}^{\alpha_{i}}$, the coherent set $A$-sphere ${ }_{i-1}$ has a new probability of ${ }^{8}$

$$
t_{i}^{\text {down }}:=\frac{\alpha_{i}}{\operatorname{Pr}(A)} \cdot \operatorname{Pr}\left(A \text {-sphere }_{i-1}\right) .
$$

If $\alpha$ is raised just very slightly above $\alpha_{i}$, then the upper $A$-sphere of $\operatorname{Pr}_{A}^{\alpha}$ is extended by $A$-layer $_{i}$ and gets a new probability of just very slightly more than $\frac{\alpha}{\operatorname{Pr}(A)} \cdot \operatorname{Pr}\left(A\right.$-sphere $\left._{i}\right)$. Let us define the limit for $\alpha$ approaching $\alpha_{i}$ from the right as

$$
t_{i}^{u p}:=\frac{\alpha_{i}}{\operatorname{Pr}(A)} \cdot \operatorname{Pr}\left(A \text {-sphere }_{i}\right) .
$$

The function specifying the posterior probability $\operatorname{Pr}_{A}^{\alpha}\left(\operatorname{Upp}_{\operatorname{lo}}\left(\operatorname{Pr}_{A}^{\alpha}\right)\right)$ of the upper $A$-sphere in dependence of $\alpha$ has jump discontinuities at each $\alpha_{i}$ for which $n_{i} \neq 0$ and $p_{i} \neq 0$. The term $\left.\frac{\operatorname{Pr}(A \text {-sphere }}{i}\right) \cdot \alpha$ gives exactly the probability of the upper $A$-sphere after Jeffrey conditionalisation by $A$ with $\alpha$, provided that $\alpha$ is in the interval $\left(\alpha_{i}, \alpha_{i+1}\right]$, for $i=1, \ldots, r-1$. If $\alpha \leq \alpha_{1}$, then the upper $A$-sphere is empty, and we may put the corresponding probability to 0 . If $\alpha>\alpha_{r}$, then the value given by (4) reduces to $\alpha$ and the posterior probability of the upper $A$-sphere is $\alpha$. It makes good sense to define $\alpha_{0}=0$ and $\alpha_{r+1}=1$. So we obtain

$\operatorname{Pr}_{A}^{\alpha}\left(\operatorname{Upp} A\left(\operatorname{Pr}_{A}^{\alpha}\right)\right)=\frac{\operatorname{Pr}\left(A \text {-sphere }_{i}\right)}{\operatorname{Pr}(A)} \cdot \alpha$, for the $i \in\{0, \ldots, r\}$ such that $\alpha \in\left(\alpha_{i}, \alpha_{i+1}\right]$.

This (set of) equation(s) represents the posterior probability of the upper $A$-sphere (of the posterior probability function) as a piecewise-defined function of $\alpha$.

The greater the Jeffrey parameter $\alpha$, the greater the relevant index $i$ is in (5), and therefore the greater the value given by (5). In other words, (5) specifies a monotonically increasing function of $\alpha$. As we have mentioned, this function is not continuous, it has a jumps at each $\alpha_{i}$ for which $n_{i} \neq 0$ and $p_{i} \neq 0$. Between the jumps, the function consists of sections of linear functions of the form const $_{i} \cdot \alpha$.

If the value given in (5) is at least as big as the threshold parameter $t$, then $\operatorname{Pr}_{A}^{\alpha}$ accepts $A$ at threshold value $t$, and the corresponding belief set $\operatorname{Bel}_{t}\left(\operatorname{Pr}_{A}^{\alpha}\right)$ is a subset of $A$. This we know from Observation 7.

\footnotetext{
${ }^{8}$ Here and elsewhere, it makes sense to put $A$-sphere ${ }_{0}=\emptyset$. So $t_{1}^{\text {down }}=0$.
} 
Now suppose that the context sets a threshold value $t$. We need to find an $\alpha$ such that after Jeffrey conditionalisation by $A$ with $\alpha$, the posterior belief set $\operatorname{Bel}_{t}\left(\operatorname{Pr}_{A}^{\alpha}\right)$ is a subset of the proposition $A$. The minimal change condition tells us to identify an $\alpha$ that is as small as possible subject to the requirement that $A$ be accepted at the global threshold $t$.

We consider the function giving us the Jeffrey parameter $\alpha$ that is needed in order to guarantee a certain probability of the upper $A$-sphere of the updated probability measure, or more precisely, to guarantee that $\operatorname{Pr}_{A}^{\alpha}\left(\operatorname{Upp}_{A}\left(\operatorname{Pr}_{A}^{\alpha}\right)\right) \geq t$ (see again Observation 7). This means that $t$ is taken to be the independent variable (set by the context, as we proposed) and $\alpha$ is the dependent variable. So we need to look at the inverse of the function given in (5), that is: ${ }^{9}$

$$
\begin{array}{r}
\alpha(t)=\frac{\operatorname{Pr}(A)}{\operatorname{Pr}\left(A \text {-sphere }_{i}\right)} \cdot t, \text { for the } i \in\{1, \ldots, r\} \text { such that } t \in\left(t_{i}^{\text {up }}, t_{i+1}^{\text {down }}\right] \\
\text { and } \operatorname{Pr}\left(A \text {-sphere }_{i}\right) \neq 0 .
\end{array}
$$

This function assigns minimal $\alpha$ 's to given $t$ 's, but it is only partially defined in the interval $[0,1]$. It is undefined in the intervals $\left(t_{i}^{\text {down }}, t_{i}^{u p}\right]$, for all $i \in\{1, \ldots, r\}$. If the contextually given threshold $t$ lies in one of these intervals, there simply is no $\alpha$ that gives $\operatorname{Pr}_{A}^{\alpha}\left(\operatorname{Upp} A\left(\operatorname{Pr}_{A}^{\alpha}\right)\right)=t$.

There is not in general a minimal $\alpha$ which achieves acceptance of $A$ with the global threshold remaining at, or set to, $t$. Therefore the minimality intuition cannot always be satisfied. For the intervals of $t$ for which the function specified in (6) is undefined, any $\alpha$ above the relevant $\alpha_{i}$ will accept $A$ with a safety of more than $t$. However, there is a 'natural' upper limit for $\alpha$ stemming from the requirement that the resulting revision be minimising: $\alpha$ should be less than or equal to $\alpha_{i+1}$. That is, $\alpha$ should be set so that we at least do not add any more layers than needed in order to acquire the belief that $A$. As the same layers are involved every such $\alpha$ will deliver the same belief set; for if $\alpha, \alpha^{\prime} \in\left(\alpha_{i}, \alpha_{i+1}\right]$, then $\operatorname{Bel}_{t}\left(\operatorname{Pr}_{A}^{\alpha}\right)=\operatorname{Bel}_{t}\left(\operatorname{Pr}_{A}^{\alpha^{\prime}}\right)$. With only finitely many layers, the existence of minimalising revisions is guaranteed.

So at the level of the belief set, there is a unique solution to the question of what constitutes a minimal revision with $A$. If, however, a unique solution at the level of the underlying probability measure is desired, we can recommend two ways to proceed. The first one uses the idea of adding a very small 'just noticeable difference' $\varepsilon$ to $\alpha_{i}$; the second one is to take the Jeffrey parameter to be determined by some function of (and into) the interval $\left(\alpha_{i}, \alpha_{i+1}\right]$, for instance the function selecting the last element $\alpha_{i+1}$, the function $\frac{1}{2}\left(\alpha_{i}+\alpha_{i+1}\right)$ ('halfway'), or some other such function. Here are two simple recommendations for the revision recipes:

$$
\begin{array}{r}
\alpha(t)=\alpha_{i}+\varepsilon, \text { for the } i \in\{1, \ldots, r\} \text { such that } t \in\left(t_{i}^{\text {down }}, t_{i}^{u p}\right] \\
\text { (first recommendation); } \\
\alpha(t)=\alpha_{i+1}, \text { for the } i \in\{1, \ldots, r\} \text { such that } t \in\left(t_{i}^{\text {down }}, t_{i}^{u p}\right] \\
\text { (second recommendation). }
\end{array}
$$

The first recommendation is gentler and more conservative, the second does not require the introduction of a new and somewhat arbitrary magnitude of a 'just noticeable difference'.

\footnotetext{
${ }^{9}$ If $\operatorname{Pr}\left(A\right.$-sphere $\left._{i}\right)=0$, then $t_{i}^{\text {down }}=t_{i}^{u p}=0$. The case $t=0$ does not matter for (6). The case $0<t \leq t_{1}^{u p}$ is dealt with in (7) and (8).
} 
There are two problems with our recommended solutions. First, there are of course smaller values of $\alpha(t)$ that would serve the agent's aim of accepting $A$ equally well, like $\alpha(t)=\alpha_{i}+\frac{\varepsilon}{2}$ and $\alpha(t)=\frac{1}{2}\left(\alpha_{i}+\alpha_{i+1}\right)$, respectively. Second, our recommended solutions succeed in producing functions mapping any threshold $t$ to a unique $\alpha$ that does the job (which is good), but they both produce functions that are non-monotonic (which is bad). ${ }^{10}$ But since no other general solution to the task of non-minimally accepting $A$ can avoid either of these problems, we stick to our recommendations as the most natural ones. In fact we have a slight preference for the first recommendation because its $\alpha$ 's will generally be smaller than those of the second.

So the full function defining the Jeffrey parameter $\alpha$ in terms of $t$ over the whole interval $(0,1]$ is obtained by piecing together (6) and (7) (or alternatively, by piecing together (6) and (8)). In any case, $\alpha(t)$ is greater than or equal to $t$.

It should be (re)emphasised that even in those cases where there is a unique solution $\alpha$, it will not in general be the case that $\operatorname{Pr}_{A}^{\alpha}(A)=t$. While we will always have $\operatorname{Pr}_{A}^{\alpha}(A) \geq t$ sometimes the inequality will be strict. The difference $\alpha(t)-t$ may be seen as a measure, dependent on both the probability function $\mathrm{Pr}$ and the threshold $t$, of the effort involved in accepting $A$ coherently - in contrast to accepting $A$ using the Lockean thesis directly. We may call $\alpha(t)-t$ the cost of coherence. It allows that some propositions become accepted even though one is far from holding them certain, while other propositions that do not cohere as well with one's belief set are not accepted at that level, and so it allows that some beliefs are 'easier' to acquire than others, in the sense that they do not require as compelling evidence for their truth.

Starting from a probability measure $\mathrm{Pr}$ and a fixed, contextually determined threshold value $t$, an agent can implement a belief revision by transitioning to $\operatorname{Pr}_{A}^{\alpha}$, where $\alpha$ is uniquely determined by (6) and (7), or alternatively, by (6) and (8). Respecting both the coherence of the belief state and the prevailing threshold $t$, this $\operatorname{Pr}_{A}^{\alpha}$ may be regarded as the probability measure that 'accepts' $A .{ }^{11}$ Other than standard conditionalisation $\operatorname{Pr}(. \mid A)$, it does not make $A$ absolutely certain. It only makes $A$ as certain as is required by the context and coherence-i.e., certain to the degree $\alpha(t)$. As a Jeffrey conditionalisation, it is in line with Bayesian ideology. It does not need an explicit specification of $\alpha$. The Jeffrey parameter $\alpha$ is (as it were, automatically) determined by the contextually given threshold $t$.

\subsection{A six-layered example}

Suppose that the global threshold value is set to 0.75 . We can refine and extend the example depicted in Fig. 2 in order to illustrate how the Coherentist Locke-Jeffrey model works. It features a prior belief state with 18 possible worlds having six different probability values (i.e., $r=6$ ). Suppose we want to revise this state by a proposition $A$. The relevant properties of the belief state are given by 18 parameters: six for the probability levels of the individual worlds (we already know these), six for the number of $A$-worlds and six for the number of $\bar{A}$-worlds at each of the six probability levels.

Our extended example is depicted in Fig. 5. $\mathrm{Bel}_{0.75}(\mathrm{Pr})$ is the proposition consisting of the $\sum_{i=1}^{4} n_{i}$ most probable $A$-worlds and the $\sum_{i=1}^{4} m_{i}$ most probable $\bar{A}$-worlds. Obviously,

\footnotetext{
${ }^{10}$ While the function is monotonically increasing over most of its domain, we get that $\alpha\left(t_{i}^{u p}\right)$ is greater than

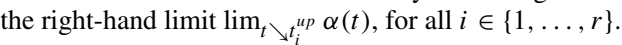

${ }^{11}$ We might thus have used the notation ' $P r * A$ ' for this $\operatorname{Pr}_{A}^{\alpha}$. We have chosen not to do this, in order to avoid introducing yet more notation.
} 


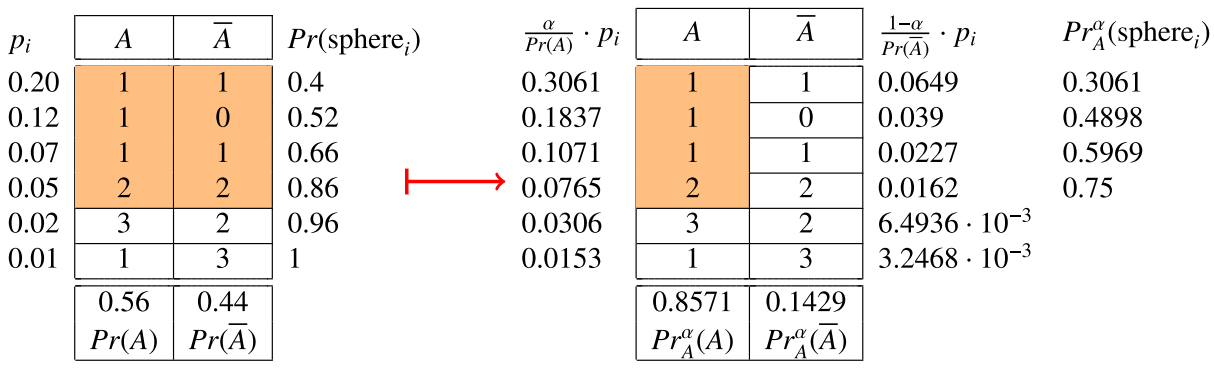

Fig. 5 The six-layered example, with $t=0.75$, and its revision 7 by $A$

this proposition is consistent with $A$. In order to have a coherent set consisting entirely of $A$ worlds and having a probability of 0.75 , we need to take $\alpha$ not lower than $0.75 \cdot \frac{\operatorname{Pr}(A)}{\sum_{i=1}^{4}\left(n_{i} \cdot p_{i}\right)}=$ $0.75 \cdot \frac{0.56}{0.49}=0.8571 .^{12}$ By the principle of minimal change, we ought to choose this $\alpha$ minimal subject to the condition that the probability of the upper $A$-sphere reaches 0.75 . So we take exactly the value $\alpha=0.8571$. The new belief set $\operatorname{Bel}_{0.75}\left(\operatorname{Pr}_{A}^{\alpha}\right)$ thus is the proposition consisting of the $\sum_{i=1}^{4} n_{i}$ most probable $A$-worlds-'probable' now according to $\operatorname{Pr}_{A}^{\alpha}$. $\operatorname{Bel}_{0.75}\left(\operatorname{Pr}_{A}^{\alpha}\right)$ here is exactly identical to $\operatorname{Bel}_{0.75}(\operatorname{Pr}) \cap A$-but we will see below that this identity does not necessarily hold.

\subsection{Belief withdrawal}

Let us now turn to belief withdrawal. Here there are cases in which no maximal solution exists. The handling of such cases needs to be slightly different from the handling of the absence of minimal solutions for belief revision. We modify (6)-(8) accordingly. The easy cases where there is a maximal solution are covered by equation

$$
\alpha(t)=\alpha_{i}, \text { for the } i \in\{1, \ldots, r\} \text { such that } t \in\left(t_{i}^{\text {down }}, t_{i}^{u p}\right]
$$

Even when there is no maximal solution, there still are solutions that minimise the effect of a withdrawal on the belief state. Here are three recommendations for such minimising withdrawals:

$$
\begin{aligned}
& \alpha(t)=\left(\frac{\operatorname{Pr}(A)}{\operatorname{Pr}\left(A \text { - } \text { phere }_{i}\right)} \cdot t\right)-\varepsilon \quad \text { (first recommendation) } \\
& \alpha(t)=\alpha_{i} \quad \text { (second recommendation) } \\
& \alpha(t)=\frac{\operatorname{Pr}(A)}{\operatorname{Pr}\left(A \text { - }^{\left.- \text {phere }_{i+1}\right)}\right)} \cdot t \quad \text { (third recommendation) }
\end{aligned}
$$

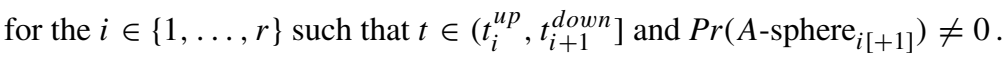

Here we tend to favour the third recommendation. In contrast to the first recommendation, it leaves the compounded function defined by (9) and (11) monotonic and it does not require an extra parameter $\varepsilon$, and its 'correction' is much gentler than the second recommendation (which provides the smallest maximising contraction).

Starting from a probability measure $P r$ and a fixed, contextually determined threshold value $t$, an agent can implement a belief withdrawal by transitioning to $\operatorname{Pr}_{A}^{\alpha}$, where $\alpha$ is

${ }^{12}$ Notice that $\frac{\alpha}{\operatorname{Pr}(A)}=1.5306, \frac{1-\alpha}{\operatorname{Pr}(\bar{A})}=0.3247$, and that $\frac{\alpha}{\operatorname{Pr}(A)} \cdot p_{4}>\frac{1-\alpha}{\operatorname{Pr}(\bar{A})} \cdot p_{1}>\frac{\alpha}{\operatorname{Pr}(A)} \cdot p_{5}$. 


\begin{tabular}{|c|c|c|c|c|c|c|c|c|}
\hline \multirow{4}{*}{$\begin{array}{l}p_{i} \\
0.375 \\
0.25\end{array}$} & & & \multirow[b]{2}{*}{$\operatorname{Pr}\left(\right.$ sphere $\left._{i}\right)$} & \multirow{2}{*}{$\frac{\alpha}{\operatorname{Pr}(A)} \cdot p_{i}$} & $A$ & $\bar{A}$ & \multirow{3}{*}{$\begin{array}{l}\frac{1-\alpha}{\operatorname{Pr}(\bar{A})} \cdot p_{i} \\
0.25 \\
0.1667\end{array}$} & $\operatorname{Pr}_{A}^{\alpha}\left(\right.$ sphere $\left._{i}\right)$ \\
\hline & $A$ & $\bar{A}$ & & & 1 & 1 & & 045 \\
\hline & 1 & 1 & 0.75 & $\begin{array}{l}0.43 \\
0.3\end{array}$ & $\begin{array}{l}1 \\
1\end{array}$ & $\frac{1}{0}$ & & 0.75 \\
\hline & 1 & 0 & 1 & & & & & 1 \\
\hline & $\begin{array}{l}0.625 \\
\operatorname{Pr}(A)\end{array}$ & $\begin{array}{l}0.375 \\
\operatorname{Pr}(\bar{A})\end{array}$ & & & $\begin{array}{c}0.75 \\
\operatorname{Pr}_{A}^{\alpha}(A)\end{array}$ & $\begin{array}{c}0.25 \\
\operatorname{Pr}_{A}^{\alpha}(\bar{A})\end{array}$ & & \\
\hline
\end{tabular}

Fig. 6 Counterexample to preservation for $t=0.75$ : It does not hold that $B e l * A \subseteq B e l$, even though $A$ is consistent with Bel. The revision is minimising

uniquely determined by (9) and (11) (or, at the reader's option, by (9) and (10), or by (9) and (11)). Respecting both the coherence of the belief state and the prevailing threshold $t$, this $\operatorname{Pr}_{A}^{\alpha}$ may be regarded as the probability measure that 'unaccepts' $A .{ }^{13}$ But it does not make $A$ absolutely improbable. It only makes $A$ as uncertain as is required by the context and coherence-in general, its probability is not even lowered to less than $t !^{14}$ No external specification of the Jeffrey parameter $\alpha$ is needed, $\alpha$ is (automatically) determined by the contextually given threshold $t$.

\section{Properties of changes of belief states}

Suppose that the categorical belief set $\mathrm{Bel}$ is derived from a probability function $\mathrm{Pr}$ and a global threshold $t$ by the equation $B e l=B_{e l}(P r)$. Then we can think of the categorical revision of $\mathrm{Bel}$ by $A$, in symbols $B e l * A$, as the belief set $\operatorname{Bel}_{t}\left(\operatorname{Pr}_{A}^{\alpha}\right)$, where $\alpha$ is determined by (6) in conjunction with a favourite recommendation for revisions, i.e., either (7) or (8). Similarly, we can construe the categorical withdrawal of $A$ from $\mathrm{Bel}$, in symbols $\mathrm{Bel} \dot{-A}$, as the belief set $\operatorname{Bel}_{t}\left(\operatorname{Pr}_{A}^{\alpha}\right)$, where $\alpha$ is determined by (9) in conjunction with a favourite recommendation for withdrawals, i.e., one of the (10), (11) and (11). We will now investigate some properties of the change operations on categorical beliefs thus defined.

\subsection{Failure of preservation and inclusion for belief revision}

The following two principles have been widely accepted in the literature on the revision of categorical beliefs.

Preservation If $A$ is consistent with $\mathrm{Bel}$, then $\mathrm{Bel} * A \subseteq B e l$.

Inclusion $\operatorname{Bel} \cap A \subseteq B e l * A$.

Syntactical counterparts of these conditions play crucial roles as 'basic' rationality postulates in the classical belief revision theory of Alchourrón, Gärdenfors and Makinson ('AGM' [2]). Judging from our six-layered example, it may seem that the Coherentist Locke-Jeffrey model satisfies Preservation and Inclusion, too. However, we will now show that it in fact does not validate them. For the sake of clarity and simplicity, the (classes of) examples given in this and the following two subsections are cases in which unique optimal-minimal or

\footnotetext{
${ }^{13}$ We might have introduced the notation ' $\mathrm{Pr} \dot{-} A$ ' for this $\operatorname{Pr}_{A}^{\alpha}$.

${ }^{14}$ See the withdrawal of $A$ in Fig. 8 below: while $t$ is 0.75 , the posterior probability of $A$ is still 0.844 . So there is no 'cost of coherence' in the case of withdrawals. On the contrary, the insistence on coherence makes it easier to eliminate a belief.
} 


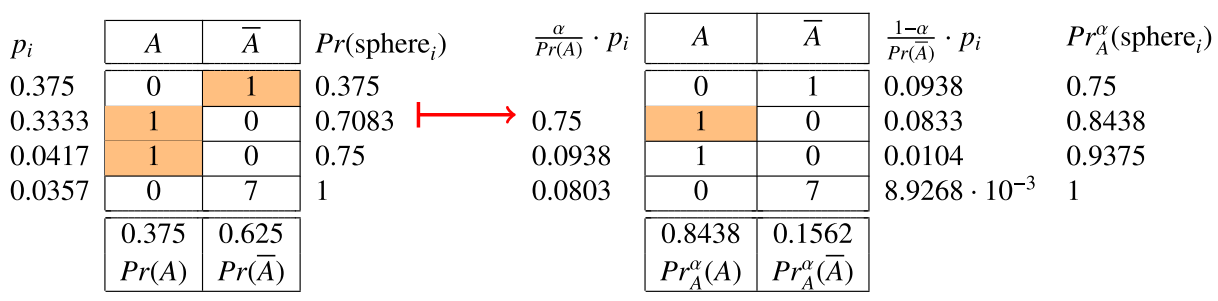

Fig. 7 Counterexample to inclusion for $t=0.75$ : It does not hold that $\mathrm{Bel} \cap A \subseteq B e l * A$. The revision is minimising

maximal-Jeffrey parameters $\alpha$ are available and we do not have to resort to any of our recommendations.

Observation 8 The Coherentist Locke-Jeffrey model for the revision of categorical beliefs does not validate Preservation. More precisely, for any threshold $t$ such that $0<t<1$ there exists a set of worlds $U$, a belief state $(\operatorname{Pr}, t)$, and a proposition $A$ such that there is no $A$-accepting revision $\mathrm{Bel} * A$, minimising or not, that satisfies Preservation.

For the proof of Observation 8 , we have chosen a very simple prior belief state with only two layers; the special case $t=0.75$ is represented in Fig. 6. However, nothing depends on that choice, more layers only give more degrees of freedom, and thus make it easier to find violations of Preservation.

Observation 9 The Coherentist Locke-Jeffrey model for the revision of categorical beliefs does not validate Inclusion. More precisely, for any threshold $t$ such that $0<t<1$ there exists a set of worlds $U$, a belief state $(\operatorname{Pr}, t)$, and a proposition $A$ such that there is no $A$-accepting revision $\mathrm{Bel} * A$, minimising or not, that satisfies Inclusion.

The special case $t=0.75$ of the construction used in the proof of Observation 9 is represented in Fig. 7.

We summarise that viewed as an operation on belief sets, the Coherentist Locke-Jeffrey model of revising by a proposition $A$ satisfies neither Inclusion nor Preservation, i.e., neither of the rather basic third and fourth AGM axioms. We think of this as a feature of this model, not as a bug.

Although Inclusion and Preservation are principles that look persuasive at first sight, they have been criticised repeatedly. The failure of Inclusion is characteristic of 'inductively extended belief revision' as modelled by Levi [20, pp. 142-151] and of 'abductive belief revision' as modelled by Pagnucco [26] and Nayak [25]. Rabinowicz [27, p. 106] even claims that violations of Inclusion (which he calls 'Conservativity') are 'quite central to our intellectual lives'. ${ }^{15}$ Preservation fails to hold for possible worlds semantics if the the possible worlds are only partially ordered with respect to their plausibility [29, p. 12]. Noteworthy arguments against Preservation are put forward in the context of Cross's [5] revision based on 'non-monotonic reasoning policies', Levi's [20, pp. 29-42, 59-65] 'Ramsey revision', Rabinowicz's [27] 'stable revision', Rott's [28, Chapter 5] 'foundational belief change', Lin and Kelly's [24] revision based on 'odds threshold acceptance rules' and Shear and Fitelson's [31] 'Lockean revision'.

\footnotetext{
${ }^{15}$ Unfortunately, Rabinowicz does not elaborate on this remark.
} 


\begin{tabular}{|c|c|c|c|c|c|c|c|c|}
\hline \multirow{2}{*}{$\begin{array}{l}p_{i} \\
0.75\end{array}$} & $A$ & $\bar{A}$ & \multirow{2}{*}{$\begin{array}{l}\operatorname{Pr}\left(\text { sphere }_{i}\right) \\
0.75\end{array}$} & \multirow{2}{*}{$\begin{array}{l}\frac{\alpha}{\operatorname{Pr}(A)} \cdot p_{i} \\
0.6881\end{array}$} & $A$ & $\bar{A}$ & \multirow[t]{2}{*}{$\frac{1-\alpha}{\operatorname{Pr}(\bar{A})} \cdot p_{i}$} & \\
\hline & 1 & 0 & & & 1 & 0 & & 0.6881 \\
\hline 0.17 & 1 & 0 & \multirow{3}{*}{$\begin{array}{l}0.92 \\
1\end{array}$} & \multirow{3}{*}{$\begin{array}{l}0.156 \\
0.0734\end{array}$} & 1 & 0 & \multirow{3}{*}{$\begin{array}{l}0.3314 \\
0.156\end{array}$} & 1 \\
\hline \multirow[t]{2}{*}{0.08} & 0 & 1 & & & 0 & 1 & & \\
\hline & $\begin{array}{c}0.92 \\
\operatorname{Pr}(A)\end{array}$ & $\begin{array}{c}0.08 \\
\operatorname{Pr}(\bar{A})\end{array}$ & & & $\begin{array}{c}0.844 \\
\operatorname{Pr}_{A}^{\alpha}(A)\end{array}$ & $\begin{array}{c}0.156 \\
\operatorname{Pr}_{A}^{\alpha}(\bar{A})\end{array}$ & & \\
\hline
\end{tabular}

Fig. 8 Counterexample to recovery for $t=0.75$ : It does not hold that $(\mathrm{Bel} \dot{-} A) \cap A \subseteq B e l$

\subsection{Satisfaction of inclusion and failure of recovery for belief withdrawal}

A very plausible condition for the withdrawal of propositions from belief sets is the following:

\section{Inclusion for withdrawals $B e l \subseteq B e l \doteq A$.}

Inclusion for withdrawals is valid in the traditional AGM model, but it has been criticized by Hansson [11] and Booth, Chopra, Ghose and Meyer [3]. In the Coherentist Locke-Jeffrey model, it is satisfied:

Observation 10 The Coherentist Locke-Jeffrey model for the withdrawal of categorical beliefs satisfies Inclusion for belief withdrawals.

The AGM theory of belief change suggests that a principle of Recovery should hold for belief withdrawals. In our context, it reads as follows:

Recovery $\quad(B e l-A) \cap A \subseteq B e l$.

Observation 11 The Coherentist Locke-Jeffrey model for the withdrawal of categorical beliefs does not validate Recovery. More precisely, for any threshold $t$ such that $0<t<1$ there exists a set of worlds $U$, a belief state $(\operatorname{Pr}, t)$, a proposition $A$ and a minimising contraction $\mathrm{Bel} \stackrel{-}{-A}$ such that $\mathrm{Bel} \stackrel{-}{-}$ A does not satisfy Recovery.

The special case $t=0.75$ of the construction used in the proof of Observation 11 is represented in Fig. 8.

So the Coherentist Locke-Jeffrey model of belief withdrawal violates Recovery, i.e., it violates the fifth AGM axiom for belief contractions. Again, we do not think that this is a bad feature of the model. The Recovery postulate has been very critically received from early on in the belief change literature (see Hansson [10] and Levi [19]).

\subsection{Failure of the Levi and Harper identities}

In standard belief revision theory, there is the well-known Levi identity connecting belief revision and belief withdrawal:

Levi identity $\quad B e l * A=(B e l-\bar{A}) \cap A$.

The so-called Harper identity is a kind of converse of the Levi identity:

Harper identity $\quad B e l-A=B e l \cup(B e l * \bar{A})$. 
In the Coherentist Locke-Jeffrey model, it is easy to come up with examples violating both the Levi and the Harper identity, the common reason being that in our model the revision $\mathrm{Bel} * \mathrm{~A}$ is not in general a subset of the withdrawal $\mathrm{Bel}-\overline{\mathrm{A}}$. We believe that this exactly as it should be. For a withdrawal of $\bar{A}$, only a few comparatively improbable $A$ worlds are sufficient to thwart the formation of a coherent and sufficiently probable belief set accepting $\bar{A}$. Much more is needed, however, for the revision by $A$, namely a coherent and sufficiently probable compass of comparatively probable $A$-worlds. Here we can see again how making the connection of coherent probability and categorical belief calls into question principles that have been regarded as eminently plausible in discussions that deal with plain belief only. Formally, we get

\section{Observation 12}

(i) The Coherentist Locke-Jeffrey model of belief change does not validate the Levi identity when minimising contraction methods are used. More precisely, for any threshold $t$ such that $0<t<1$ there exists a set of worlds $U$, a belief state $(\operatorname{Pr}, t)$, and a proposition $A$ such that there is a minimising contraction $B e l-\bar{A}$ for which no revision $B e l * A$, minimising or not, satisfies the Levi-identity with respect to $\mathrm{Bel} \dot{\bar{A}} \overline{\text {. }}$

(ii) The Coherentist Locke-Jeffrey model does not validate the Harper identity when minimising contraction methods are used. More precisely, for any threshold $t$ such that $0<t<1$ there exists a set of worlds $U$, a belief state $(\operatorname{Pr}, t)$, and a proposition $A$ such that there is a minimising contraction $\mathrm{Bel}-A$ for which no revision $\mathrm{Bel} * \overline{\mathrm{A}}$, minimising or not, satisfies the Harper identity with respect to $\mathrm{Bel} \dot{-} \mathrm{A}$.

The special case $t=0.75$ of the construction used in the proof of Observation 12(i) is represented in Fig. 9.

\begin{tabular}{|c|c|c|c|c|c|c|c|c|}
\hline \multirow{7}{*}{$\begin{array}{l}p_{i} \\
0.85 \\
0.09 \\
0.022 \\
0.019\end{array}$} & $A$ & $\bar{A}$ & \multirow{7}{*}{$\begin{array}{l}\operatorname{Pr}\left(\text { sphere }_{i}\right) \\
0.85 \\
0.94 \\
0.962 \\
1\end{array}$} & \multirow[t]{2}{*}{$\frac{\alpha}{\operatorname{Pr}(A)} \cdot p_{i}$} & $A$ & $\bar{A}$ & $\frac{1-\alpha}{\operatorname{Pr}(\bar{A})} \cdot p_{i}$ & $\operatorname{Pr}_{A}^{\alpha}\left(\right.$ sphere $\left._{i}\right)$ \\
\hline & 0 & 1 & & & 0 & 1 & \multirow{6}{*}{$\begin{array}{l}0.717 \\
0.0759 \\
0.0186 \\
0.016\end{array}$} & \multirow{6}{*}{$\begin{array}{l}0.717 \\
0.8689 \\
1\end{array}$} \\
\hline & 0 & 1 & & \multirow{5}{*}{$\begin{array}{l}0.3106 \\
0.0759 \\
0.0656\end{array}$} & 0 & 1 & & \\
\hline & 1 & 0 & & & 1 & 0 & & \\
\hline & 2 & 0 & & & 2 & 0 & & \\
\hline & 0.06 & 0.94 & & & 0.2071 & 0.7929 & & \\
\hline & $\operatorname{Pr}(A)$ & $\operatorname{Pr}(\bar{A})$ & & & $\operatorname{Pr}_{A}^{\alpha}(A)$ & $\operatorname{Pr}_{A}^{\alpha}(\bar{A})$ & & \\
\hline \multirow{7}{*}{$\begin{array}{l}p_{i} \\
0.85 \\
0.09 \\
0.022 \\
0.019\end{array}$} & A & $\bar{A}$ & \multirow{7}{*}{\multicolumn{2}{|c|}{$\begin{array}{l}\operatorname{Pr}\left(\text { sphere }_{i}\right) \\
0.85 \\
0.94 \\
0.962 \\
1\end{array}$}} & $A$ & $\bar{A}$ & \multirow{7}{*}{$\begin{array}{l}\frac{1-\alpha}{\operatorname{Pr}(\bar{A})} \cdot p_{i} \\
0.2261 \\
0.0239 \\
5.8509 \cdot 10^{-3} \\
5.0531 \cdot 10^{-3}\end{array}$} & $\operatorname{Pr}_{A}^{\alpha}\left(\right.$ sphere $\left._{i}\right)$ \\
\hline & 0 & 1 & & & 0 & 1 & & \multirow{6}{*}{$\begin{array}{l}0.275 \\
0.75 \\
0.9761 \\
1\end{array}$} \\
\hline & 0 & 1 & & & 0 & 1 & & \\
\hline & 1 & 0 & & & 1 & 0 & & \\
\hline & 2 & 0 & & & 2 & 0 & & \\
\hline & 0.06 & 0.94 & & & 0.75 & 0.25 & & \\
\hline & $\operatorname{Pr}(A)$ & $\operatorname{Pr}(\bar{A})$ & & & $\operatorname{Pr}_{A}^{\alpha}(A)$ & $\operatorname{Pr}_{A}^{\alpha}(\bar{A})$ & & \\
\hline
\end{tabular}

Fig. 9 Counterexample to the Levi identity for $t=0.75$. The upper part shows $B e l \dot{-} \bar{A}$, the lower part $B e l * A$. It does not hold that $\mathrm{Bel} * A \subseteq(\mathrm{Bel}-\bar{A}) \cap A$. Both the contraction and the revision are minimising 


\section{Discussion}

The probabilistic notion of a coherent belief set is not new to this paper. For instance, it is discussed by Cantwell [4] in relation to categorical belief. But it can also be linked to other proposals.

Lin and Kelly [23] have proposed what they call the odds threshold rule or the camera shutter rule of acceptance. Changing terminology somewhat and simplifying the formal apparatus, they basically propose that a belief set $\mathrm{Bel}$ should be based on a threshold $s$ in the open interval $(0,1)$ in the following way:

Odds Threshold Rule $\quad \operatorname{Bel}_{S}(\operatorname{Pr})=\left\{v: \frac{\operatorname{Pr}(v)}{\max _{u \in U} \operatorname{Pr}(u)}>1-s\right\}$.

As the camera shutter rule is based on pair-wise comparisons between worlds and worlds with maximal probability, it is easy to see that every belief set generated by the camera shutter rule will be coherent, and conversely, that every coherent set can be generated by the camera shutter rule for some $r$. This means that the notion of a coherent belief set is extensionally identical to Lin and Kelly's notion of an acceptance set based on the camera shutter rule.

Although extensionally equivalent we think the approach of the present paper provides a better motivation for the resulting space of coherent belief sets (possible acceptance sets); at the very least it gives a different motivation. For in the present paper the notion of a coherent belief set has been characterised independently of any acceptance rule. Furthermore, the static acceptance rule itself - choose a coherent belief set that exceeds the contextually given threshold $t$-has a more natural 'ring' to it than a threshold based on pairwise comparisons of possible worlds. However, the most striking difference lies in the dynamics. While Lin and Kelly only use strict Bayesian conditionalisation, we have opted for Jeffrey conditionalisation which is much more in line with the idea of belief short of probability 1 .

The notion of a coherent set includes the notion of what Leitgeb [17] has dubbed $r$-stable sets of worlds. ${ }^{16}$ A proposition $A$ is $r$-stable (with $r \geq 0.5$ ) if and only if for all propositions $B$ such that $\operatorname{Pr}(B)>0$ and $B$ is consistent with $A, \operatorname{Pr}(A \mid B)>r$. In a finite setting, a proposition $A$ is $r$-stable if and only if for every $u \in A, \operatorname{Pr}(u)>\frac{r}{1-r} \cdot \sum_{v \notin A} \operatorname{Pr}(v)$, that is, if and only if every world in $u$ is more probable than the sum of the probabilities of all non- $A$ worlds, scaled with a factor that depends on the threshold $r$. Clearly any stable set will be a coherent belief set on the present proposal. The converse, however, does not hold: coherent belief sets need not be stable in Leitgeb's sense.

So Leitgeb's notion of a stable belief set is more restrictive than the current notion of a coherent belief set. As only coherent belief sets with probability greater than 0.5 can be stable one could interpret this more restrictive approach as a consequence of taking belief to be closed under logical commitments (so the belief set itself counts as a belief and as such should have a probability above 0.5 ). However, on the present approach there are many coherent sets with probability greater than 0.5 that still fail to be stable, ${ }^{17}$ so the more restrictive nature of Leitgeb's notion of a stable set cannot be wholly accounted for by taking belief to be closed under logical commitments.

Leitgeb's Humean Thesis requires rational belief sets to be $r$-stable, for some $r \geq 0.5$. It draws on the seemingly plausible idea that learning (by Bayesian conditioning) something

\footnotetext{
${ }^{16}$ More precisely, he calls such sets $\operatorname{Pr}$-stable ${ }^{r}$.

${ }^{17}$ For instance, let $W=\left\{w_{1}, w_{2}, w_{3}, w_{4}\right\}$ and $\operatorname{Pr}\left(w_{1}\right)=0.6, \operatorname{Pr}\left(w_{2}\right)=0.2$ and $\operatorname{Pr}\left(w_{3}\right)=\operatorname{Pr}\left(w_{4}\right)=0.1$. Then $\left\{w_{1}, w_{2}\right\}$ is a coherent set (and it has probability 0.8 ) but it is not a stable set.
} 
new that is consistent with what one believes should not make one's beliefs unduly improbable. That is, it is a requirement on 'static' belief that draws on the plausibility of a property similar to Preservation in the dynamics of belief. But this very feature can be questioned.

Consider the following example. A coin is about to be tossed six times. One is nearly certain (probability 0.99) that it is a fair, well-balanced coin, but there is a very small chance (probability 0.01) that it is a fake coin that always lands heads. Consider a partition which contains a world for every possible sequence of tosses given that the coin is fair and a world in which one gets six heads and the coin is fake. The probability that the coin is fair and that one will get a particular sequence of outcomes is roughly 0.015 . The probability that the coin is unfair and that one will get a particular sequence of outcomes is either 0.01 (if the outcomes are all heads) or 0 (if not). The proposition that the coin is fair is thus a 0.5 -stable proposition and has a probability of 0.99: a plausible candidate for being a proposition that one can reasonably accept.

Say now that we learn that the coin landed heads all six times, which is perfectly consistent with the proposition accepted. The probability of this happening given that it is a fair coin is roughly 0.016 , the probability of it happening given that it is a fake coin is 1 . Conversely, the probability of it being a fair coin given that it lands heads six times is roughly 0.6 , and that it is fake is roughly 0.4 . The proposition that it is a fair coin is still a 0.5 stable proposition, but now with a modest 0.6 probability. The relatively 'low' probability suggests that it would be reasonable to then surrender the belief that the coin is fair, even though the outcome was consistent with what one initially accepted. If this is a reasonable scenario it would undermine the contention that stability is an adequacy criterion for belief: some outcomes that are consistent with what one believes can, if they occur, seriously lessen the likelihood of the belief being true to the extent that it undermines the grounds for acceptance. $^{18}$

Interestingly, in another paper Lin and Kelly [24] study what happens when the dynamics of categorical beliefs (expansion, revision and contraction) can be taken to be driven by changes on an underlying probabilistic measure. They study strict conditionalisation and find that if categorical belief change is to track probabilistic belief change, it is not possible (on pain of triviality) to satisfy Preservation. Our results in Section 6.1 to some extent corroborate and extend these results, as they show-based on Definition 2 rather than on OTR - that when the underlying dynamics of categorical belief is driven by Jeffrey conditionalisation, one can expect both Inclusion and Preservation to fail. (We remind the reader that all our results are restricted to the finite case.)

\section{Conclusion}

We have combined four ideas for belief state change, each of which is very simple: the Lockean thesis, coherence, Jeffrey conditionalisation and minimal change. The resulting model shows a fairly complex behaviour. It is, of course, highly idealised and makes some strong assumptions. The most problematic assumption is probably the reliance on a partition. But as indicated in Section 3, there may be ways of getting rid of this partition-reliance, and some philosophers actually think of it as an advantage (Yalcin [33]). Now let us recap some of the main advantages of the present model.

\footnotetext{
${ }^{18}$ One could object that if a global probability threshold of 0.6 is too low for belief, the stability threshold should be set higher, e.g., require that a proper belief set should be 0.9 -stable. But then the stability requirement would not allow that one initially believes that the coin is fair, even though its probability is very high (0.99).
} 
Consider first the static level. We have shown how a probability measure combined with a global threshold allows one to extract a probabilistically informative consistent belief state as soon as the probability measure is (positively) non-uniform; this is done by selecting the strongest coherent belief set with a probability exceeding a contextually given global threshold. The beliefs supported by such a state are jointly consistent and closed under consequences (including, of course, conjunction). We have also shown that the closure of a consistent belief base that is generated by the Lockean Thesis (using a local threshold that is high enough to avoid contradictions as in the lottery paradox) is a coherent belief state, thereby linking the Lockean Thesis to the notion of coherence. Furthermore, when one 'iterates' the Lockean Thesis - taking the belief state generated by the Lockean Thesis to be given and applying the Lockean thesis again - the consistent belief states generated in this fashion are all coherent. Indeed, all coherent belief states can be generated in this way.

We have thus achieved a simple and harmonious pairing of an agent's (categorical, plain) belief with her degrees of belief as represented by a subjective probability function, and shown how it connects to the Lockean Thesis. Categorical belief can be had short of probability one. Global thresholds - thresholds regulating the whole belief state - need not be 'high'; the values have in fact remained formally unrestricted in this paper (except that they must not be zero). Indeed, low global thresholds enable us to explain what is happening in (one version of) the paradox of the preface. We have shown how one can combine a low global threshold with a high (iteratively applied) local threshold.

Consider next the dynamics. In the Coherentist Locke-Jeffrey model, the plain belief change operation 'Update your belief state by $A$ ' is interpreted as the injunction to believe $A$ to a degree that surpasses a threshold (the threshold can either be a low global threshold or a high local threshold). Belief changes operate on the probability functions in the first place. They are fully Bayesian in spirit since they are first of all Jeffrey conditionalisations. Like Locke, Jeffrey leaves room for belief below maximal certainty. There is nothing new about this, of course; what is new in our model is that we determine (or at least formulate constraints on) a Jeffrey parameter $\alpha$ in just such a way that is suitable for acquiring the belief $A$ - given that the global threshold value in operation remains the same. The parameter does not have to be specified either by the evidence or by the subject's feelings of certainty. We have also shown how to apply the same idea for the withdrawal of beliefs.

It has turned out that the Coherentist Locke-Jeffrey model violates quite a number of postulates that have been considered as fundamental for belief revision and contraction in the classical AGM tradition-to wit, Preservation, Inclusion, Recovery and the Levi and Harper Identities. We don't think that this is a genuine problem. In our view, a good constructive model almost always overrides postulates that have been put up in abstracto, ${ }^{19}$ and our model indeed helps us understand how the relevant postulates can come to fail if plain beliefs are somehow grounded in degrees of belief.

Can we characterise the conditions under which the traditional belief change postulates fail, or can we perhaps find weaker versions of them that are still valid? Our short answer is 'no'. Finding the classes of counterexamples (one for each $t$ between 0 and 1 ) in the proofs of Observations 8-12 was sometimes easy, sometimes hard. But in no case did we find an intuitively perspicuous and compactly characterisable condition specifying where the postulates remain valid and where not. This is due to the considerable complexities in the interaction of sizeable numbers of threshold values, probability values and of possible

\footnotetext{
${ }^{19}$ This is argued for in Rott [29]. However, that article defends Preservation (and Inclusion) for belief revisions in a basic propositional language - a position that we feel is getting increasingly eroded, at least when plain beliefs may be understood as founded on credences. Witness the papers by Lin and Kelly [23, 24] and by Shear and Fitelson [31] as well as the present paper.
} 
worlds. We have grown the conviction that the operations we have presented are sui generis. They teach us that it is wrong from the start to expect the traditional postulates to hold if the set of categorical beliefs is, as it were, only the tip of an iceberg of a belief state including degrees of belief (or default assumptions/expectations for that matter). What happens to this 'visible' part is not determined by this very part alone, but by the structure underneath. The postulates of classical belief revision theories create the impression that there are simple set-theoretic (logical) relations between prior and posterior beliefs, and between revisions and contractions. But we submit that this is an illusion. If changes operate on the structure underneath, the changes in the visible part can be explained, but they are not as simple as if the underlying structure did not exist.

Assuming that a certain threshold is contextually given, we have modelled the acquisition or retraction of a categorical belief by purely Bayesian means. The threshold value need not even be explicitly mentioned in the revision process. But the Coherentist Locke-Jeffrey model allows for a rich variety of changes of belief states. First, ordinary Jeffrey conditionalisation of the probability function by $A$ with an externally specified safety parameter $\alpha$ may of course be retained as a direct Bayesian operation on the probability component of belief states.

Second, threshold values may be set and reset by the context-adjusting the threshold may perhaps even be interpreted as a special type of belief revision operation. In acknowledging that different threshold values may be operative or relevant in different situations, we acknowledge that belief is a highly context-dependent and/or vague notion. Importantly, however, the model is prepared to keep things apart: epistemic changes due to new evidence (updating with a fixed threshold) are distinguished from pragmatic changes due to new circumstances or contexts (varying the threshold).

Third, as a more complex belief change operation, the acquisition of a belief with a certain safety parameter that differs from the threshold currently in operation can be modelled. This may be done by first resetting the threshold to a new value $t^{\prime}$ and then accepting $A$ with this threshold. The reverse operation of first accepting $A$ and then resetting the threshold generally gives a different result and does not necessarily lead to the acceptance of $A$. There is an order-dependence of the final belief state, the operations of resetting the threshold and accepting a proposition are not commutative.

Fourth, iterations of belief changes can be performed without any problems. They are just iterations of Jeffrey conditionalisations. And such conditionalisations with parameters short of 1 avoid posterior zero probabilities of contingent propositions (i.e., they avoid irregular probability functions) that might cause problems at later steps in a sequence of belief changes.

Acknowledgements We gratefully acknowledge the generous support by the Swedish Collegium for Advanced Study in Uppsala where both authors enjoyed ideal research conditions as fellows in spring 2016. We also thank audiences at the 2017 Madeira Workshop on Belief Revision, Argumentation, Ontologies and Norms, at the 7th annual meeting of the DFG priority program New Frameworks of Rationality in Etelsen and at a workshop on New Perspectives on Conditionals and Reasoning in Regensburg, as well as two anonymouos reviewers of this journal for excellent questions and comments.

Open Access This article is distributed under the terms of the Creative Commons Attribution 4.0 International License (http://creativecommons.org/licenses/by/4.0/), which permits unrestricted use, distribution, and reproduction in any medium, provided you give appropriate credit to the original author(s) and the source, provide a link to the Creative Commons license, and indicate if changes were made. 


\section{Appendix: Proofs}

Proof of Observation 2 (i) Assume that Bel is consistent and locally threshold based. So there is some threshold $t$ such that $\mathrm{Bel}=\bigcap\{C: \operatorname{Pr}(C) \geq t\}$. Assume that $u \in \mathrm{Bel}$ and that $\operatorname{Pr}(u) \leq \operatorname{Pr}(v)$. As $u \in \operatorname{Bel}, \operatorname{Pr}(\overline{\{u\}})<t$. As $\operatorname{Pr}(u) \leq \operatorname{Pr}(v)$, $\operatorname{Pr}(\overline{\{v\}}) \leq \operatorname{Pr}(\overline{\{u\}})$. So $\operatorname{Pr}(\overline{\{v\}})<t$. As $\overline{\{v\}}$ is the weakest proposition incompatible with $v$ we know that for every $C$ such that $\operatorname{Pr}(C) \geq t, v \in C$. So $v \in B e l$.

(ii) Assume that $\operatorname{Pr}$ assumes at least two different values on $U$. Let $D$ be the set of $P r$ minimal worlds in $U$ (i.e. $u \in D$ iff $\operatorname{Pr}(u) \leq \operatorname{Pr}(v)$ for all $v \in U)$. Let $t=1-\operatorname{Pr}(u)$, where $u \in D$. Let $B e l=\bar{D}$. One can show that

$$
\mathrm{Bel}=\bigcap\{C: \operatorname{Pr}(C) \geq t\},
$$

i.e. that $\mathrm{Bel}$ is threshold based. For note that $\operatorname{Pr}(\overline{\{u\}}) \geq t$ for all $u \in D$. This means that for all $u \in D, u \notin \bigcap\{C: \operatorname{Pr}(C) \geq t\}$. So $\bigcap\{C: \operatorname{Pr}(C) \geq t\} \subseteq B e l$. Assume that $v \in \operatorname{Bel}(=\bar{D})$. As $\operatorname{Pr}(v)>\operatorname{Pr}(u)($ for $u \in D), 1-\operatorname{Pr}(v)<t$. As $\operatorname{Pr}(\overline{\{v\}})=1-\operatorname{Pr}(v)$, $\operatorname{Pr}(\overline{\{v\}})<t$. But $\overline{\{v\}}$ is the weakest proposition excluding $v$, so $v \in C$ for each $C$ such that $\operatorname{Pr}(C) \geq t$. So $v \in \bigcap\{C: \operatorname{Pr}(C) \geq t\}$ and so $B e l \subseteq \bigcap\{C: \operatorname{Pr}(C) \geq t\}$.

(iii) Assume that $\operatorname{Pr}$ assumes at least two different positive values on $U$. Now let $E$ be the set of positive $\operatorname{Pr}$-minimal worlds (i.e. $u \in E$ iff $\operatorname{Pr}(u) \neq 0$ and $\operatorname{Pr}(u) \leq \operatorname{Pr}(v)$ for all $v \in U$ such that $\operatorname{Pr}(v) \neq 0)$. Let $t=1-\operatorname{Pr}(u)$ where $u \in E$. Where $D$ as before is the set of $\operatorname{Pr}$-minimal worlds, let $\mathrm{Bel}=\overline{\mathrm{D} \cup \mathrm{E}}$. Clearly, $0<\operatorname{Pr}(\mathrm{Bel})<1$, and one can show that

$$
B e l=\bigcap\{C: \operatorname{Pr}(C) \geq t\},
$$

i.e. that $\mathrm{Bel}$ is threshold based. For note that $\operatorname{Pr}(\overline{\{u\}}) \geq t$ for all $u \in D \cup E$. This means that for all $u \in D \cup E, u \notin \bigcap\{C: \operatorname{Pr}(C) \geq t\}$. So $\bigcap\{C: \operatorname{Pr}(C) \geq t\} \subseteq B e l$. Assume that $v \in \operatorname{Bel}(=\overline{D \cup E}$ ). As $\operatorname{Pr}(v)>\operatorname{Pr}(u)$ (for $u \in E), 1-\operatorname{Pr}(v)<t$. As $\operatorname{Pr}(\overline{\{v\}})=1-\operatorname{Pr}(v), \operatorname{Pr}(\overline{\{v\}})<t$. But $\overline{\{v\}}$ is the weakest proposition excluding $v$, so $v \in C$ for each $C$ such that $\operatorname{Pr}(C) \geq t$. So $v \in \bigcap\{C: \operatorname{Pr}(C) \geq t\}$ and so $B e l \subseteq \bigcap\{C: \operatorname{Pr}(C) \geq t\}$.

Proof of Observation 3 (i) From Observation 2 we know that the threshold based belief sets form an inclusion chain. As $U$ is finite this inclusion chain has an inclusion minimal set: the strongest threshold based belief set.

(ii) Let $C$ be the strongest threshold based belief set and let $\mathrm{Bel}$ be a coherent belief set such that $C \subseteq B e l$. Let $t_{0}$ be a threshold that generates $C$ (so $C=\bigcap\{E: \operatorname{Pr}(E) \geq$ $\left.t_{0}\right\}$ ). If $\mathrm{Bel}=U$ then $\mathrm{Bel}$ is threshold based, so assume that $\mathrm{Bel} \subset U$. Let $u$ be an element of $\overline{B e l}$ such that for every element $v$ of $\overline{B e l}, \operatorname{Pr}(v) \leq \operatorname{Pr}(u)$. Let $t=1-\operatorname{Pr}(u)$. It follows that $t \geq t_{0}$ (as otherwise $u \in C$ ). As $u \notin C$ there is a proposition $E$ such that $u \notin E$ and $\operatorname{Pr}(E) \geq t_{0}$. It follows that for every proposition $E$ such that $E=\overline{\{v\}}$ where $v \notin B e l, \operatorname{Pr}(E) \geq t_{0}$, so $C \subseteq E$. Note that if $\operatorname{Pr}(E) \geq t$, then $B e l \subseteq E$ (as every element $v$ of $\mathrm{Bel}$ has a higher probability than $u$, we have $\operatorname{Pr}(\overline{\{v\}})<t$ ). So $B e l=\bigcap\{E: \operatorname{Pr}(E) \geq t\}$.

Proof of Observation 4 Let $\left\{u_{1}, \ldots, u_{n}\right\}=\overline{B e l}$. For any $u_{i}$ define $A_{i}=\overline{\left\{u_{i}\right\}}$. As $u_{i} \notin B e l$, $\operatorname{Pr}\left(u_{i}\right)<0.5$, so $\operatorname{Pr}\left(A_{i}\right)>0.5$. So there is some $t$ such that $t>0.5$ and $\operatorname{Pr}\left(A_{i}\right)>t$ for each $i$. Clearly, $\mathrm{Bel}=\bigcap_{1 \leq i \leq n} A_{i}$. 
Proof of Observation 5 Let $\mathrm{Bel}_{0}, \ldots, \mathrm{Bel}_{n}$ be an enumeration of the coherent belief sets in increasing strength.

If $n=0$, the claim is trivial. So assume that $n \geq 1$, and let $P_{B e l_{i}}()=.P\left(. \mid\right.$ Bel $\left._{i}\right)$ for any $i<n$. As $B e l_{i+1}$ is a strict subset of $B e l_{i}$ there are worlds $u$ and $v$ such that $v \in \mathrm{Bel}_{i+1}, u \notin \mathrm{Bel}_{i}$ and $u, v \in \mathrm{Bel}_{i}$ such that $\operatorname{Pr}(u)<\operatorname{Pr}(v)$. As a result $\operatorname{Pr}_{\mathrm{Bel}_{i}}(u)<$ $\operatorname{Pr}_{B e l_{i}}(v)$. Note that $\mathrm{Bel}_{i+1}$ is the maximal coherent and $\mathrm{Pr}_{B e l_{i}}$-informative belief set. From Observation 2(iii) we know that there is a $P_{B e l_{i}}$-informative locally threshold based belief set, and from Observations 2(i) and 3 we know that in relation to $\operatorname{Pr}_{B e l_{i}}, B_{e l+1}$ is threshold based.

Proof of Observation 6 Let $\mathrm{Bel}_{0}, \ldots, \mathrm{Bel}_{n}$ be an inclusion chain of consistent belief sets such that $B e l_{0}$ is locally threshold based and each $B e l_{i+1}$ is locally threshold based relative to $\mathrm{Pr}_{\mathrm{Bel}_{i}}$. Proof by induction over $n$. For $i=0$ it follows from Observation 2 that $\mathrm{Bel}_{0}$ is coherent. Assume, for the induction step, that $B e l_{i}$ is coherent. By assumption $B e l_{i+1}$ is locally threshold based relative to $\mathrm{Pr}_{\mathrm{Bel}_{i}}$. From Observations 2 and 3 we know that any threshold based belief set is coherent. So $\mathrm{Bel}_{i+1}$ is coherent given $\mathrm{Pr}_{\mathrm{Bel}_{i}}$. But as $\mathrm{Bel}_{i}$ is coherent relative to $\mathrm{Pr}, \mathrm{Bel}_{i+1}$ will also be coherent relative to $\mathrm{Pr}$.

Proof of Observation 8 For any arbitrary threshold $t$ such that $0<t<1$, we can use the following model with $W=\left\{w_{1}, w_{2}, w_{3}, \ldots, w_{n_{2}+2}\right\}$ :

\begin{tabular}{|c|c|c|}
\cline { 2 - 3 }$p_{1}$ & $A$ & $\bar{A}$ \\
\cline { 2 - 3 }$p_{2}$ & $w_{1}$ & $w_{2}$ \\
\cline { 2 - 3 }${ }_{2}$ & $w_{3}, \ldots, w_{n_{2}+2}$ & - \\
\cline { 2 - 3 } & &
\end{tabular}

So $A=\left\{w_{1}, w_{3}, \ldots, w_{n_{2}+2}\right\}, \operatorname{Pr}(A)=1-p_{1}$ and $\operatorname{Pr}(\bar{A})=p_{1}$. Let $n_{2}=$ floor $\left(\frac{2(1-t)}{t}\right)+$ $1>\frac{2(1-t)}{t}$. Notice that $n_{2}$ depends on $t$. Then we assign the following probabilities

$$
p_{1}=\frac{t}{2} \quad p_{2}=\frac{1-t}{n_{2}} .
$$

Notice that $p_{2}<\frac{(1-t) \cdot t}{2(1-t)}=\frac{t}{2}=p_{1}$ and that $2 p_{1}+n_{2} p_{2}=1$.

The belief set supported by $(\operatorname{Pr}, t)$ is $\mathrm{Bel}=\operatorname{Bel}_{t}(\mathrm{Pr})=\left\{w_{1}, w_{2}\right\}$.

Now we show that $B e l * A=\operatorname{Bel}_{t}\left(\operatorname{Pr}_{A}^{\alpha}\right)=A=\left\{w_{1}, w_{3}, \ldots, w_{n_{2}+2}\right\}$ where $\alpha$ is any arbitrary Jeffrey parameter suitable for obtaining $A$ as a belief.

Our proof strategy in choosing (13) is to have the relationship between $p_{1}$ and $p_{2}$ so that there is no $\alpha$ such that $\operatorname{Pr}_{A}^{\alpha}\left(\left\{w_{1}\right\}\right) \geq t$. This implies that even when $A$ is probable enough to be believed, the probability of $w_{1}$ can't itself pass the threshold $t$. So $w_{3}, \ldots, w_{n_{2}+2}$ will all be elements of the new belief set, which will be $A$, resulting in a violation of Preservation.

Meanwhile $\operatorname{Bel} \cap A=\left\{w_{1}\right\} \neq \emptyset$. In order for a revision by $A$ to satisfy Preservation the new belief set should be a subset of (and in fact identical to) $\left\{w_{1}\right\}$. This means that we need an $\alpha$ such that

$$
\operatorname{Pr}_{A}^{\alpha}\left(\left\{w_{1}\right\}\right) \geq t .
$$

That is, we need an $\alpha$ between 0 and 1 such that

$$
\frac{\alpha}{\operatorname{Pr}(A)} \cdot \operatorname{Pr}\left(\left\{w_{1}\right\}\right) \geq t \text {. }
$$

That is

$$
\alpha \operatorname{Pr}\left(w_{1}\right) \geq t \operatorname{Pr}(A) .
$$


But this is impossible since one can show that

$$
\operatorname{Pr}\left(w_{1}\right)<t \operatorname{Pr}(A) .
$$

This is so because we have $\operatorname{Pr}\left(w_{1}\right)=\frac{t}{2}$ and $\operatorname{Pr}(A)=1-\frac{t}{2}$, and $t\left(1-\frac{t}{2}\right)-\frac{t}{2}=\frac{1}{2} t(1-t)$ which is positive for $t$ between 0 and 1 .

So given the threshold $t$ and the probability measure $\operatorname{Pr}$ (defined in dependence of $t$ ), every possible revision of $\mathrm{Bel}=\left\{w_{1}, w_{2}\right\}$ by $A$, minimal or otherwise, results in the belief set $B e l * A=A$ and violates Preservation.

Proof of Observation 9 For any arbitrary threshold $t$ such that $0<t<1$, we can use the following model with $W=\left\{w_{1}, w_{2}, w_{3}, w_{4}, \ldots, w_{m_{4}+3}\right\}$ :

\begin{tabular}{|c|c|c|}
\cline { 2 - 3 } & $A$ & $\bar{A}$ \\
\cline { 2 - 3 }$p_{1}$ & - & $w_{1}$ \\
\cline { 2 - 3 }$p_{2}$ & $w_{2}$ & - \\
\cline { 2 - 3 }$p_{3}$ & $w_{3}$ & - \\
\cline { 2 - 3 }$p_{4}$ & - & $w_{4}, \ldots, w_{m_{4}+3}$ \\
\cline { 2 - 3 } & &
\end{tabular}

So $A=\left\{w_{2}, w_{3}\right\}$. Let $m_{4}=$ floor $\left(\frac{2(3-t)}{t}\right)+1>\frac{2(3-t)}{t}$. Notice that $m_{4}$ depends on $t$. The role of the set $\left\{w_{4} \ldots, w_{m_{4}+3}\right\}$ is to store the residual probability in such a way that it spreads so thin (over so many worlds) that it is not relevant. Then we assign the following probabilities

$$
\begin{aligned}
p_{1} & =\frac{t}{2} & p_{2} & =\frac{t}{3-t} \\
p_{3} & =\frac{t \cdot(1-t)}{2 \cdot(3-t)} & p_{4} & =\frac{1-t}{m_{4}} .
\end{aligned}
$$

One quickly finds that the assignments in (14) satisfy the requirement $p_{1}>p_{2}>p_{3}>$ $p_{4}$. Our proof strategy in choosing these assignments was to guarantee that $p_{1}+p_{2}+p_{3}=$ $t$. So the belief set supported by $(\operatorname{Pr}, t)$ is $\mathrm{Bel}=\mathrm{Bel}_{t}(\mathrm{Pr})=\left\{w_{1}, w_{2}, w_{3}\right\}$. Meanwhile $\operatorname{Pr}(A)=\frac{t}{2}$ and $\operatorname{Pr}(\bar{A})=1-\frac{t}{2}$.

The relationship between $p_{1}, p_{2}$ and $p_{3}$ is made to be such that as soon as $\operatorname{Pr}_{A}^{\alpha}\left(w_{3}\right)>$ $\operatorname{Pr}_{A}^{\alpha}\left(w_{1}\right)$, i.e., $\alpha>\alpha_{3}$, we already have $\operatorname{Pr}_{A}^{\alpha}\left(w_{2}\right) \geq t$. This implies that as soon as $A$ is probable enough to be believed, the probability of $w_{2}$ by itself passes the threshold $t$, leaving $w_{3}$ outside of the new belief set that we get when revising by $A$. Now we show that $B e l * A=\operatorname{Bel}_{t}\left(\operatorname{Pr}_{A}^{\alpha}\right)=A=\left\{w_{2}\right\}$ where $\alpha$ is any arbitrary Jeffrey parameter suitable for obtaining $A$ as a belief. Consider

$$
\begin{aligned}
\alpha_{3}=\frac{p_{1} \cdot \operatorname{Pr}(A)}{p_{3} \cdot \operatorname{Pr}(\bar{A})+p_{1} \cdot \operatorname{Pr}(A)} & =\frac{\frac{t}{2} \cdot \frac{t}{2}}{\frac{t(1-t)}{2(3-t)} \cdot \frac{2-t}{2}+\frac{t}{2} \cdot \frac{t}{2}} \\
& =\frac{t(3-t)}{(1-t)(2-t)+t(3-t)} \\
& =\frac{t(3-t)}{2} .
\end{aligned}
$$

For $\alpha=\alpha_{3}, \operatorname{Upp} A\left(\operatorname{Pr}_{A}^{\alpha}\right)=\left\{w_{2}\right\}$ and $\operatorname{Pr}\left(U p p A\left(\operatorname{Pr}_{A}^{\alpha}\right)\right)=\frac{\alpha}{\operatorname{Pr}(A)} \cdot p_{2}=\frac{t(3-t) \cdot 2 \cdot t}{2 \cdot t \cdot(3-t)}=t$. 
As long as $\alpha<\alpha_{3}, A$ is not yet believed, since then $\operatorname{Upp} A\left(\operatorname{Pr}_{A}^{\alpha}\right) \subseteq\left\{w_{2}\right\}$ and $\operatorname{Pr}\left(\left\{w_{2}\right\}\right)<$ $t$. If $\alpha>\alpha_{3}$, on the other hand, $\operatorname{Pr}\left(\left\{w_{2}\right\}\right)>t$, so $B e l * A=\left\{w_{2}\right\}$ for every $A$-accepting revision $\mathrm{Bel} * A$, minimal or otherwise.

But $\operatorname{Bel} \cap A=\left\{w_{1}, w_{2}, w_{3}\right\} \cap\left\{w_{2}, w_{3}\right\}=\left\{w_{2}, w_{3}\right\}$. So the result is a failure of Inclusion.

Proof of Observation 10 The claim is trivial if $A$ is not believed in $(P r, t)$. So suppose that $A$ is believed in $(\operatorname{Pr}, t)$ in the first place, i.e., that $\operatorname{Pr}(\operatorname{Upp} A(\operatorname{Pr})) \geq t$, or equivalently, that $i(t)<i(\bar{A})$ for the initial probability measure $\operatorname{Pr}$.

First we note that in order to withdraw the belief $A$, we need to find an $\alpha$ such that $\operatorname{Bel}_{t}\left(\operatorname{Pr}_{A}^{\alpha}\right)$ includes some $\bar{A}$-worlds. By coherence, then, it includes at least $\bar{A}$-layer $_{i(\bar{A})}$.

Second, we convince ourselves that $\alpha_{i(t)}$ is such a Jeffrey parameter $\alpha$. Let $P r^{\prime}=$ $\operatorname{Pr}_{A}^{\alpha_{i(t)}}$. Clearly, $U p p A\left(\operatorname{Pr}^{\prime}\right)$ is $A$-sphere ${ }_{i(t)-1}=$ sphere $_{i-1}$ (these are the spheres of $\operatorname{Pr}$ ). Since $i(t)<i(\bar{A})$, we know that $\alpha_{i(t)}<\operatorname{Pr}(A)$. So $\operatorname{Pr}^{\prime}(A$-sphere si(t)-1 $)=$ $\frac{\alpha_{i(t)}}{\operatorname{Pr}(A)} \cdot \operatorname{Pr}\left(A\right.$-sphere $\left._{i(t)-1}\right)<\operatorname{Pr}\left(A\right.$-sphere $\left._{i(t)-1}\right)<t$. By the definition of $\alpha_{i(t)}$, $A$-sphere ${ }_{i(t)-1}$ remains the $i-1$ st sphere of $P r^{\prime}$, and the $i$ th layer of $P r^{\prime}$ is $A$-layer ${ }_{i(t)} \cup$ $\bar{A}$-layer $_{i(\bar{A})}$. Since $A$-sphere $_{i(t)}=A$-sphere $_{i(t)-1} \cup A$-layer $_{i(t)}$, we know that the smallest sphere of $P r^{\prime}$ with a probability of at least $t$ includes $A$-sphere ${ }_{i(t)}$.

If $\alpha_{i(t)}$ is the maximal Jeffrey parameter $\alpha$ that withdraws the belief $A$, then we have proved exactly what we needed to prove.

Suppose, however, that there is a larger $\alpha>\alpha_{i(t)}$ that withdraws $A$. Then the posterior probabilites of the elements of $A$-layer $_{i(t)}$ are $\frac{\alpha}{\operatorname{Pr}(A)} \cdot p_{i(t)}>\frac{\alpha_{i(t)}}{\operatorname{Pr}(A)} \cdot p_{i(t)}$, while those of the elements of $\bar{A}$-layer $_{i(\bar{A})}$ are $\frac{1-\alpha}{\operatorname{Pr}(\bar{A})} \cdot p_{i(\bar{A})}<\frac{1-\alpha_{i(t)}}{\operatorname{Pr}(\bar{A})} \cdot p_{i(\bar{A})}$. Hence, by the definition of $\alpha_{i(t)}$, the posterior probabilites of the elements of $A$-layer $_{i(t)}$ are higher than those of the elements of $\bar{A}$-layer ${ }_{i(\bar{A})}$. Since we noted above that $\operatorname{Bel}_{t}\left(\operatorname{Pr}_{A}^{\alpha}\right)$ includes $\bar{A}$-layer ${ }_{i(\bar{A})}$, it follows by coherence, that $B_{e} l_{t}\left(\operatorname{Pr}_{A}^{\alpha}\right)$ also includes $A$-layer ${ }_{i(t)}$ and $A$-sphere ${ }_{i(t)-1}$, the elements of

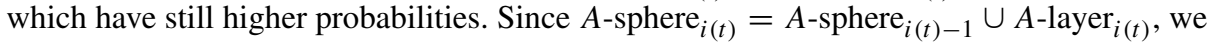
have proved exactly what we needed to prove.

Proof of Observation 11 For any arbitrary threshold $t$ such that $0<t<1$, we can use the following model with $W=\left\{w_{1}, w_{2}, \ldots, w_{n_{2}+1}, w_{n_{2}+2}, \ldots, w_{2 n_{2}+1}\right\}$ :

\begin{tabular}{c|c|c|}
\cline { 2 - 3 } & $A$ & $\bar{A}$ \\
\cline { 2 - 3 }$p_{1}$ & $w_{1}$ & - \\
\cline { 2 - 3 }$p_{2}$ & $w_{2}, \ldots, w_{n_{2}+1}$ & - \\
\cline { 2 - 3 }$p_{3}$ & - & $w_{n_{2}+2}, \ldots, w_{n_{2}+m_{3}+1}$ \\
\cline { 2 - 3 } & &
\end{tabular}

where $n_{2}=m_{3}$. So $A=\left\{w_{1}, w_{2}, \ldots, w_{n_{2}+1}\right\}$. Clearly, $\operatorname{Pr}(A)=p_{1}+n_{2} p_{2}$ and $\operatorname{Pr}(\bar{A})=$ $n_{2} p_{3}$. Let $n_{2}=m_{3}=$ floor $\left(\frac{2 \cdot(1-t)}{3 t}\right)+1>\frac{2 \cdot(1-t)}{3 t}$. Notice that this number depends on $t$. Then we assign the following probabilities

$$
p_{1}=t \quad p_{2}=\frac{2 \cdot(1-t)}{3 n_{2}} \quad p_{3}=\frac{1-t}{3 n_{2}} .
$$

Since $t=\frac{2 \cdot(1-t)}{3 \cdot 2 \cdot(1-t) / 3 t}>p_{2}$, one quickly finds that the assignments in (15) satisfy the requirement $p_{1}>p_{2}>p_{3}$. Our proof strategy in choosing these assignments was to have $p_{1}+n_{2} p_{2}+n_{2} p_{3}=1$. Clearly the belief set supported by $(\operatorname{Pr}, t)$ is $B e l=\operatorname{Bel}_{t}(\operatorname{Pr})=\left\{w_{1}\right\}$. We also get that $\operatorname{Pr}(A)=t+\frac{2}{3} \cdot(1-t)=\frac{1}{3} \cdot(2+t)$ and $\operatorname{Pr}(\bar{A})=\frac{1}{3} \cdot(1-t)$. 
The relationship between $p_{1}, p_{2}$ and $p_{3}$ is made to be such that $A$ gets lost as a belief exactly when $\operatorname{Pr}_{A}^{\alpha}\left(w_{2}\right)=\operatorname{Pr}_{A}^{\alpha}\left(w_{n_{2}+2}\right)$, i.e., when $\alpha$ is lowered to $\alpha_{2}$. This implies that as soon as $A$ is improbable enough not to be believed, the probability of $w_{2}$ by itself passes the threshold $t$, leaving $w_{3}$ outside of the new belief set that we get when revising by $A$. Now we show that $B e l-A=\operatorname{Bel}_{t}\left(\operatorname{Pr}_{A}^{\alpha}\right)=W$ where $\alpha$ is the maximal Jeffrey parameter to remove $A$ from the agent's beliefs. Consider

$$
\begin{aligned}
\alpha_{2}=\frac{p_{3} \cdot \operatorname{Pr}(A)}{p_{2} \cdot \operatorname{Pr}(\bar{A})+p_{3} \cdot \operatorname{Pr}(A)} & =\frac{\frac{1-t}{3 n_{2}} \cdot \frac{1}{3}(2+t)}{\frac{2(1-t)}{3 n_{2}} \cdot \frac{1}{3}(1-t)+\frac{1-t}{3 n_{2}} \cdot \frac{1}{3}(2+t)} \\
& =\frac{2+t}{2(1-t)+2+t} \\
& =\frac{2+t}{4-t} .
\end{aligned}
$$

As long as $\alpha>\alpha_{2}, \operatorname{Upp} A\left(\operatorname{Pr}_{A}^{\alpha}\right)=\left\{w_{1}, w_{2}, \ldots, w_{n_{2}+1}\right\}=A$ and $\operatorname{Pr}_{A}^{\alpha}\left(\operatorname{Upp} A\left(\operatorname{Pr}_{A}^{\alpha}\right)\right)=$ $\alpha>\frac{2+t}{4-t}$. But $\frac{2+t}{4-t}>t$ for $t$ between 0 and 1 (note that $\frac{2+t}{4-t}>t$ transforms to $(1-t)(2-t)>$ $0)$. So $\operatorname{Pr}\left(U p p A\left(\operatorname{Pr}_{A}^{\alpha}\right)\right)>t$ which means that $B e l=A$ as long as $\alpha_{2}<\alpha<\operatorname{Pr}(A)$.

$A$ gets lost as a belief if $\alpha \leq \alpha_{2}$. To see this, note that $\operatorname{Upp} A\left(\operatorname{Pr}_{A}^{\alpha_{2}}\right)=\left\{w_{1}\right\}$ and

$$
\operatorname{Pr}_{A}^{\alpha_{2}}\left(U \operatorname{Pp} A\left(\operatorname{Pr}_{A}^{\alpha_{2}}\right)\right)=\frac{\alpha_{2}}{\operatorname{Pr}(A)} \cdot p_{1}=\frac{2+t}{4-t} \cdot \frac{3}{2+t} \cdot t=\frac{3 t}{4-t}
$$

and this is less than $t$ for $t$ between 0 and 1 (note that $\frac{3 t}{4-t}<t$ transforms to $t(t-1)<0$ ). Thus the maximal Jeffrey parameter to eliminate $A$ from the agent's belief set is $\alpha_{2}$, and this means that the minimising $\mathrm{Bel}-A=\operatorname{Bel}_{t}\left(\operatorname{Pr}_{A}^{\alpha_{2}}\right)$ equals $W$.

But then $(\operatorname{Bel}-A) \cap A=A=\left\{w_{1}, w_{2}, \ldots, w_{n_{2}+1}\right\}$ while $B e l=\left\{w_{1}\right\}$. So the result is a failure of Recovery.

Proof of Observation 12 (i) For any arbitrary threshold $t$ such that $0<t<1$, we can use the following model with $W=\left\{w_{1}, w_{2}, w_{3}, w_{4}, \ldots, w_{n_{4}+3}\right\}$ :

\begin{tabular}{c|c|c|}
\cline { 2 - 3 } & $A$ & $\bar{A}$ \\
\cline { 2 - 3 }$p_{1}$ & - & $w_{1}$ \\
\cline { 2 - 3 }$p_{2}$ & - & $w_{2}$ \\
\cline { 2 - 3 }$p_{3}$ & $w_{3}$ & - \\
\cline { 2 - 3 }$p_{4}$ & $w_{4}, \ldots, w_{n_{4}+3}$ & - \\
\cline { 2 - 3 } & &
\end{tabular}

So $A=\left\{w_{3}, w_{4}, \ldots, w_{m_{4}+3}\right\}$. Let $n_{4}=$ floor $\left(\frac{2-t}{t}\right)+1>\frac{2-t}{t}$. Notice that $n_{4}$ depends on $t$. (The number $n_{4}$ of possible worlds at the fourth layer must be chosen greater than $\frac{2-t}{t}$, in order to make sure that $p_{4}<p_{3}$.) Then we assign the following probabilities

$$
\begin{array}{ll}
p_{1}=\frac{t \cdot(2-t) \cdot(4+t)}{6-t} & p_{2}=\frac{t \cdot(2-t) \cdot(2-2 t)}{6-t} \\
p_{3}=\frac{t \cdot(1-t)^{2}}{2} & p_{4}=\frac{1}{n_{4}} \cdot \frac{(2-t) \cdot(1-t)^{2}}{2} .
\end{array}
$$

It is routine to check that the assignments in (16) satisfy the requirements that $p_{1}>p_{2}>$ $p_{3}>p_{4}$ and that $p_{1}+p_{2}+p_{3}+n_{4} p_{4}=1$. Notice also that with the probabilities of (16), $\operatorname{Pr}(A)=(1-t)^{2}$ and $\operatorname{Pr}(\bar{A})=t \cdot(2-t)$. 
As our last bit of preparation, we verify that $\mathrm{Bel}=\mathrm{Bel}_{t}(\mathrm{Pr})=\left\{w_{1}\right\}$. For this it is sufficient to show that

$$
\frac{t \cdot(2-t) \cdot(4+t)}{6-t} \geq t
$$

But (17) reduces to $2-t(1+t) \geq 0$ which is true for all $t$ between 0 and 1 .

Now we show that $(B e l-A) \cap A=\operatorname{Bel}_{t}\left(\operatorname{Pr}_{A}^{\alpha}\right) \cap A=\left\{w_{3}\right\}$ where $\alpha$ is the minimising Jeffrey parameter to remove $\bar{A}$ from the agent's beliefs, and at the same time $B e l * A=$ $\operatorname{Bel}_{t}\left(\operatorname{Pr}_{A}^{\alpha}\right)=\left\{w_{3}, w_{4}, \ldots, w_{n_{4}+3}\right\}$ where $\alpha$ is any Jeffrey parameter suitable for obtaining $A$ as a belief.

We now turn to revisions. Given the definition of $p_{3}$ it follows that the upper $A$-layer is never sufficient for accepting $A$, as the proportion of this layer within the proposition $A$ is less than $t: \frac{p_{3}}{\operatorname{Pr}(A)}<t$. So in any revision by $A$, even for $\alpha=1$, the resulting belief set will include the worlds $\left\{w_{4}, \ldots, w_{n_{4}+3}\right\}$ of the fourth layer.

Next contractions. We are assuming that when there is a minimising way of contracting $\bar{A}$ (a maximal Jeffrey parameter yielding a contraction) this is the unique contraction method that will be used. We want to establish (a) that there exists a minimal way of contracting $\bar{A}$, and (b) that the resulting belief set does not contain the worlds from the fourth layer. Once this is shown we will have a violation of the Levi identity.

We first establish that there is a minimal way of contracting $\bar{A}$. We do this in two steps. First we show that as long as the world $w_{2}$ of the second layer is more probable than the world $w_{3}$ of the third layer the first and second layer jointly exceed the threshold (which means that $\bar{A}$ is believed). That is, as long as $\frac{\alpha}{\operatorname{Pr}(A)} \cdot p_{3}<\frac{1-\alpha}{\operatorname{Pr}(\bar{A})} \cdot p_{2}$, we have $t \leq 1-\alpha$. Simplifying the first expression we thus need to show that as long as

$$
\alpha t<(1-\alpha) \cdot \frac{4-4 t}{6-t}
$$

we have $t \leq 1-\alpha$. Now (18) is equivalent to

$$
t \cdot \frac{6-t}{4+2 t-t^{2}}<1-\alpha .
$$

So it is enough to show that

$$
1 \leq \frac{6-t}{4+2 t-t^{2}}
$$

After a few transformations, (20) reduces to $t(3-t) \leq 2$ which is quickly seen to hold for $t$ between 0 and 1 .

Next we show that as soon as the third layer becomes as probable as the second layer, $\bar{A}$ is not believed any more; that is, as soon as $\frac{\alpha}{\operatorname{Pr}(A)} \cdot p_{3}=\frac{1-\alpha}{\operatorname{Pr}(\bar{A})} \cdot p_{2}$ we have $\frac{1-\alpha}{\operatorname{Pr}(\bar{A})} \cdot p_{1}<t$ (when the first layer falls below the threshold we need more layers but now the worlds in the original layers 2 and 3 are equiprobable, which means we have to add $A$-worlds to the belief set to get above the threshold which means that $\bar{A}$ is withdrawn). Simplifying the first expression we get

which is equivalent to

$$
\alpha t=(1-\alpha) \cdot \frac{4-4 t}{6-t}
$$

$$
t \cdot \frac{6-t}{4+2 t-t^{2}}=1-\alpha .
$$

What we need to show is:

$$
(1-\alpha) \cdot \frac{4+t}{6-t}<t .
$$


For this it is sufficient to show that

$$
t \cdot \frac{6-t}{4+2 t-t^{2}}<t \cdot \frac{6-t}{4+t},
$$

or equivalently $\frac{1}{4+t(2-t)}<\frac{1}{4+t}$ which clearly holds for all $t$ between 0 and 1 .

Finally we show that when $\alpha$ is chosen to ensure a minimal contraction of $\bar{A}$, the fourth layer is not in the resulting belief set. That is, when $\frac{\alpha}{\operatorname{Pr}(A)} \cdot p_{3}=\frac{1-\alpha}{\operatorname{Pr}(\bar{A})} \cdot p_{2}$, the sum of the three top layers (that is, $\left.\frac{1-\alpha}{\operatorname{Pr}(\bar{A})} \cdot p_{1}+\frac{1-\alpha}{\operatorname{Pr}(\bar{A})} \cdot p_{2}+\frac{\alpha}{\operatorname{Pr}(A)} \cdot p_{3}=(1-\alpha)+\frac{\alpha}{\operatorname{Pr}(A)} \cdot p_{3}\right)$ is greater or equal to $t$. Simplifying the expressions we need to show that

$$
(1-\alpha)+\frac{\alpha t}{2} \geq t
$$

which reduces to

$$
1-\alpha \geq \frac{t}{2-t}
$$

Now we can make use of (22) and see that it is sufficient to show that

$$
t \cdot \frac{6-t}{4+2 t-t^{2}} \geq \frac{t}{2-t} .
$$

After a few transformations, (27) reduces to $t(5-t) \leq 4$ which is easily recognised to be true for all $t$ between 0 and 1 .

(ii) To see that the Harper identity fails we use the same model as above, with the roles of $A$ and $\bar{A}$ reversed. Then we have $B e l=\left\{w_{1}\right\} \subseteq A, B e l-A=\left\{w_{1}, w_{2}, w_{3}\right\}$ and $B e l * \bar{A}=$ $\left\{w_{3}, w_{4}, \ldots, w_{n_{4}+3}\right\}$. Thus neither Bel-A$\subseteq$ Bel $\cup B e l * \bar{A}$ nor Bel $\cup B e l * \bar{A} \subseteq B e l \dot{A}$, and the Harper identity is doubly violated.

\section{References}

1. Adler, J.E.: Belief's Own Ethics. MIT Press, Cambridge (2002)

2. Alchourrón, C.E., Gärdenfors, P., Makinson, D.: On the logic of theory change: partial meet contraction and revision functions. J. Symb. Log. 50(2), 510-530 (1985)

3. Booth, R., Chopra, S., Ghose, A., Meyer, T.: Belief liberation (and retraction). Stud. Logica. 79, 47-72 (2005)

4. Cantwell, J.: Static justification in the dynamics of belief. Erkenntnis 50(2-3), 481-503 (1999)

5. Cross, C.B.: Belief revision, nonmonotonic reasoning, and the Ramsey test. In: Kyburg, H.E., Loui, R.P., Carlson, G.N. (eds.) Knowledge Representation and Defeasible Reasoning, pp. 223-244. Kluwer, Boston (1990)

6. Diaconis, P., Zabell, S.L.: Updating subjective probability. J. Am. Stat. Assoc. 77(380), 822-830 (1982)

7. Dietrich, F., List, C., Bradley, R.: Belief revision generalized: a joint characterization of Bayes's and Jeffrey's rules. J. Econ. Theory 162, 352-371 (2016)

8. Douven, I.: Assertion, knowledge, and rational credibility. Philos. Rev. 115(4), 449-485 (2006)

9. Grove, A.: Two modellings for theory change. J. Philos. Log. 17(2), 157-170 (1988)

10. Hansson, S.O.: Belief contraction without recovery. Stud. Logica. 50(2), 251-260 (1991)

11. Hansson, S.O.: Ten philosophical problems in belief revision. J. Log. Comput. 13, 37-49 (2003)

12. Jeffrey, R.C.: Contributions to the Theory of Inductive Probability. PhD thesis Princeton University (1957)

13. Jeffrey, R.C.: The Logic of Decision, 2nd edn. University of Chicago Press, Chicago (1983)

14. Kaplan, M.: Believing the improbable. Philos. Stud. 77(1), 117-146 (1995)

15. Kyburg, H.E. Jr.: Probability and the Logic of Rational Belief. Wesleyan University Press, Middletown (1961)

16. Lehrer, K.: Theory of Knowledge. Routledge, London (1990)

17. Leitgeb, H.: The Humean thesis on belief. Proc. Aristot. Soc. Suppl. Vol. 89(1), 143-185 (2015) 
18. Leitgeb, H.: The Stability of Belief: How Rational Belief Coheres with Probability. Oxford University Press, Oxford (2017)

19. Levi, I.: The Fixation of Belief and its Undoing: Changing Beliefs Through Inquiry. Cambridge University Press, Cambridge (1991)

20. Levi, I.: For the Sake of Argument: Ramsey Test Conditionals, Inductive Inference, and Nonmonotonic Reasoning. Cambridge University Press, Cambridge (1996)

21. Levi, I.: Pragmatism and change of view. Can. J. Philos. 28(sup1):177-201, 1998. Reprinted n I. L., Pragmatism and Inquiry: Selected Essays. Oxford University Press, Oxford (2012)

22. Lewis, D.: Counterfactuals. Blackwell, Oxford (1973)

23. Lin, H.I., Kelly, K.T.: A geo-logical solution to the lottery paradox, with applications to conditional logic. Synthese 186(2), 531-575 (2012)

24. Lin, H., Kelly, K.T.: Propositional reasoning that tracks probabilistic reasoning. J. Philos. Log. 41(6), 957-981 (2012)

25. Nayak, A.: Acceptance without minimality. In: Ojeda-Aciego, M., de Guzmán, I.P., Brewka, G., Pereira, L.M. (eds.) Logics in Artificial Intelligence. Proceedings of the European Workshop (JELIA 2000), LNAI 1919, pp. 163-178. Springer, Berlin (2000)

26. Pagnucco, M.: The Role of Abductive Reasoning Within the Process of Belief Revision. PhD thesis, Basser Department of Computer Science University of Sydney (1996)

27. Rabinowicz, W.: Stable revision, or is preservation worth preserving? In: Fuhrmann, A., Rott, H. (eds.) Logic, Action, and Information: Essays on Logic in Philosophy and Artificial Intelligence, pp. 101-128. de Gruyter, Berlin (1996)

28. Change, H.R.: Choice and Inference: a Study in Belief Revision and Nonmonotonic Reasoning. Oxford University Press, Oxford (2001)

29. Preservation, H.R.: Postulation lessons from the new debate on the Ramsey test. Mind 126(502), 609626 (2017)

30. Rott, H., Pagnucco, M.: Severe withdrawal (and recovery). J. Philos. Log. 28(5), 501-547 (1999)

31. Shear, T., Fitelson, B.: Two approaches to belief revision. Erkenntnis, 2018. Online 8 February 2018 (2018)

32. van Benthem, J.: Logic and the flow of information. In: Prawitz, D., Skyrms, B., Westerståhl, D. (eds.) Logic, Methodology and Philosophy of Science IX: Proceedings of the Ninth International Congress of Logic, Methodology and Philosophy of Science (Uppsala 1991), pp. 693-724. Elsevier, Amsterdam (1993)

33. Yalcin, S.: Belief as question-sensitive. Philos. Phenomenol. Res. 97(1), 23-47 (2018)

Publisher's note Springer Nature remains neutral with regard to jurisdictional claims in published maps and institutional affiliations. 